\title{
Relationship of MMP-14 and TIMP-3 Expression with Macrophage Activation and Human Atherosclerotic Plaque Vulnerability
}

\author{
Jason L. Johnson, ${ }^{1,2}$ Nicholas P. Jenkins, ${ }^{1,2}$ Wei-Chun Huang, \\ Karina Di Gregoli, ${ }^{1,2}$ Graciela B. Sala-Newby, ${ }^{2}$ Vincent P. W. Scholtes, ${ }^{3}$ \\ Frans L. Moll, ${ }^{3}$ Gerard Pasterkamp, ${ }^{3}$ and Andrew C. Newby ${ }^{2}$ \\ ${ }^{1}$ Laboratory of Cardiovascular Pathology, School of Clinical Sciences, Level 7, Bristol Royal Infirmary, Bristol BS2 8HW, UK \\ ${ }^{2}$ School of Clinical Sciences, University of Bristol, Bristol BS2 8HW, UK \\ ${ }^{3}$ University Medical Centre, 3584 Utrecht, The Netherlands
}

Correspondence should be addressed to Jason L. Johnson; jason.l.johnson@bris.ac.uk

Received 31 March 2014; Revised 9 July 2014; Accepted 21 July 2014; Published 24 August 2014

Academic Editor: Beatriz De las Heras

Copyright ( 2014 Jason L. Johnson et al. This is an open access article distributed under the Creative Commons Attribution License, which permits unrestricted use, distribution, and reproduction in any medium, provided the original work is properly cited.

\begin{abstract}
Matrix metalloproteinase-14 (MMP-14) promotes vulnerable plaque morphology in mice, whereas tissue inhibitor of metalloproteinases-3 (TIMP-3) overexpression is protective. MMP-14 ${ }^{\text {hi }}$ TIMP- $3^{\text {lo }}$ rabbit foam cells are more invasive and more prone to apoptosis than MMP-14 ${ }^{\text {lo }}$ TIMP- $3^{\text {hi }}$ cells. We investigated the implications of these findings for human atherosclerosis. In vitro generated macrophages and foam-cell macrophages, together with atherosclerotic plaques characterised as unstable or stable, were examined for expression of MMP-14, TIMP-3, and inflammatory markers. Proinflammatory stimuli increased MMP-14 and decreased TIMP-3 mRNA and protein expression in human macrophages. However, conversion to foam-cells with oxidized LDL increased MMP-14 and decreased TIMP-3 protein, independently of inflammatory mediators and partly through posttranscriptional mechanisms. Within atherosclerotic plaques, MMP-14 was prominent in foam-cells with either pro- or antiinflammatory macrophage markers, whereas TIMP-3 was present in less foamy macrophages and colocalised with CD206. MMP14 positive macrophages were more abundant whereas TIMP-3 positive macrophages were less abundant in plaques histologically designated as rupture prone. We conclude that foam-cells characterised by high MMP-14 and low TIMP-3 expression are prevalent in rupture-prone atherosclerotic plaques, independent of pro- or anti-inflammatory activation. Therefore reducing MMP-14 activity and increasing that of TIMP-3 could be valid therapeutic approaches to reduce plaque rupture and myocardial infarction.
\end{abstract}

\section{Introduction}

Plaque rupture accounts for three quarters of myocardial infarctions [1]. Weakening of the fibrous cap owing to net degradation of extracellular matrix is believed to play an important part in plaque rupture [2]. Foam-cell macrophages (FCMs) are an abundant source of several matrix metalloproteinases (MMPs) in human plaques [3,4]. Protective effects of certain MMPs on smooth muscle migration and proliferation have been identified from experimental studies but high levels of MMP production, especially from FCMs, have been implicated in plaque progression and rupture [2]. Attention has focused recently on MMP-14, which is an activated membrane type MMP, and a tissue inhibitor of MMPs (TIMP3 ), which binds to the extracellular matrix. Given these properties, MMP-14 and TIMP-3 are likely to play important divergent roles in the regulation of macrophage pericellular proteolysis. Adoptive transfer of leukocytes lacking MMP-14 into LDL-receptor null mice increased plaque collagen content, implying a harmful collagen-depleting role of MMP-14 in plaque stability [5]. Conversely, transgenic overexpression of TIMP-3 in mouse macrophages reduced atherosclerosis formation and improved markers of plaque stability [6]. A MMP- $14^{\text {hi }}$ TIMP- $^{\text {lo }}$ subpopulation of rabbit FCMs was 
shown to have greater proteolytic capacity, ability to invade through synthetic extracellular matrix (ECM), and greater propensity to undergo apoptosis, all of which would be expected to favour plaque rupture [7]. MMP- $14^{\text {hi }}$ TIMP- $3^{\text {lo }}$ FCMs were identified in human atherosclerotic plaques [7] but the mechanisms responsible for generating the MMP- $14^{\text {hi }}$ TIMP- ${ }^{\text {lo }}$ FCMs and their relationship to plaque vulnerability in man were not previously investigated.

Macrophages can express a spectrum of different activation states dependent on pro- and anti-inflammatory signals $[8,9]$, although the effects on MMP-14 and TIMP-3 expression are not fully documented. For example, activation by Toll-like receptor agonists [10] and other inflammatory cytokines acting through nuclear factor- $\kappa \mathrm{B}(\mathrm{NF}-\kappa \mathrm{B})$ upregulates several MMPs [11]. Such activated macrophages have been associated with unstable plaque morphology, whereas deactivated or alternatively activated macrophages are associated with increased plaque stability in most [1214], although not all, studies [15]. Phenotypes resulting from oxidised phospholipids [16] or by the action of haem at sites of thrombosis and intraplaque haemorrhage have also been recently distinguished [17-19].

To clarify the nature and role of MMP- $14^{\text {hi }}$ TIMP- $3^{\text {lo }}$ FCMs we investigated their activation in vitro with pro- and anti-inflammatory molecules. We then investigated whether macrophage populations with opposing expression of MMP14 and TIMP-3 colocalise with markers of macrophage activation and correlate with histological markers of vulnerability to rupture in human carotid atherosclerotic plaques.

\section{Materials and Methods}

2.1. Carotid Artery Specimens. Carotid endarterectomy specimens came from the AtheroExpress Biobank, the details of which have been described in detail elsewhere [20-22]. Briefly, patient demographics including cardiovascular risk factors, previous medication use, and presenting symptoms were recorded. Atherosclerotic plaques were dissected by a dedicated technician into $5 \mu \mathrm{m}$ thick cross-sectional segments. Histological staining (haematoxylin and eosin (HE), Picrosirius Red (PS)) were performed on the so-called culprit lesion (segment with largest plaque area). The area occupied by following parameters was scored semiquantitatively as described previously [20-22]: atheroma core (HE, PS), calcification, (HE), collagen (PS), smooth muscle cells ( $\alpha \mathrm{SM}$ ctin; M0851; Dako), and macrophages (CD68; M0814; Dako) (Table 1). Smooth muscle cells and macrophages were also scored quantitatively by using a microscope equipped with a digital camera and using AnalySIS 3.2 software (Soft Imaging System GmbH, Munster, Germany). The amount of microvessels was quantified by using an anti-CD34 antibody; details have been described previously [20-22]. As specified in the AtheroExpress protocol, histological sections were classified according to overall appearance into: "atheromatous lesions" containing a large lipid core $(>40 \%$ of plaque area), high macrophage infiltration with low smooth muscle cell and collagen content, "fibrous lesions" with a small $(<40 \%)$ or absent lipid core, low macrophage content and high smooth muscle cell and collagen content, and "fibrousatheromatous lesions" as an intermediate between the two other phenotypes. The inter- and intraobserver variability for this classification has been described previously [23]. Adjacent sections were then taken for the histological analyses reported here, which were performed and analysed by 2 additional operators who were blinded as to the lesion classification.

2.2. Immunohistochemistry. Sections were dewaxed in Clearene and rehydrated in absolute alcohol. After rinsing in distilled water, slides were subjected to heat-induced antigen retrieval by incubation in citrate buffer $(10 \mathrm{mM}$ citric acid in $1 \mathrm{x}$ PBS, $\mathrm{pH}$ 6.0) and microwaved for 10 minutes at full power. Slides were allowed to cool for 30 minutes and then washed with PBS $(3 \times 2$ minutes). For brightfield or fluorescence IHC, $50 \mu \mathrm{L}$ of $1 \%$ bovine serum albumin or Image-iT FX Signal Enhancer (Invitrogen, Life Technologies, Paisley, UK), respectively, was placed on sections and incubated at room temperature for 30 minutes. The solution was then removed by tapping the slides and $50 \mu \mathrm{L}$ of the applicable primary antibody (as detailed in Table 1) added to the samples and incubated either overnight at $4^{\circ} \mathrm{C}$ or for 1 hour at room temperature. Sections were then washed in PBS $(3 \times 2$ minutes each $)$ and incubated in the desired secondary antibody as detailed in Table 1. For fluorescent labelling, sections were incubated in the dark for 1 hour at room temperature. Sections were then washed in PBS $(3 \times 2$ minutes) and mounted with ProLong Gold antifade reagent containing DAPI (Invitrogen, Life Technologies, Paisley, UK) to identify the nuclei. A negative control where the primary antibody was replaced with the relevant species IgG at the same dilution was always included and the cells were counted in $6 \times 20$ magnification fields. Positive cells were counted and expressed as a percentage of total nucleated cells. The percentage of CD68 positive macrophages that were also positively stained for other proteins of interest was counted by comparing serial sections or after dualimmunohistochemistry if the antibodies were suitable, by independent observers (JLJ, NPJ, VWPS) and the results averaged, as described previously [24].

For dual fluorescence, primary antibodies from different species were used and incubated together. Subsequently, species-specific fluorophore conjugated secondary antibodies were used to yield either a red fluorescent product at the site of the antigen (AlexaFluor 594) or a green fluorescent product at the site of the antigen (AlexaFluor 488). Sections were then mounted in ProLong Gold Antifade Reagent with 4',6diamidino-2-phenylindole (Life Technologies, Paisley, UK; P-36931) to fluorescently label nuclei blue. The specificity of the immunolabeling was demonstrated by inclusion of a negative control using isotype-specific nonimmune serum or IgG. Images were acquired from a fluorescence microscope using ImageProPlus image analysis software (Media Cybernetics) and merged if required before further analysis. The percentage of MMP-14 positive macrophages that stained for other proteins was counted within each lesion and expressed as percentage of total MMP-14 positive macrophages. 
Table 1: Antibodies used for immunohistochemistry.

\begin{tabular}{lcccccc}
\hline Antibody & Supplier & Cat. no. & Species & Dilution (Western) & Dilution (IHC) & Secondary antibody \\
\hline Arginase-1 & Santa Cruz & sc-20150 & Rabbit & n/a & $1 / 50$ & Goat anti-rabbit \\
CD206 & R\&D & AF2534 & Goat & $1 / 100$ & $1 / 50$ & Chicken anti-goat \\
CD68 & Dako & M0876 & Mouse & n/a & $1 / 100$ & Chicken anti-mouse \\
COX-2 & Abcam & AB15191 & Rabbit & $1 / 2000$ & $1 / 200$ & Goat anti-rabbit \\
MMP-14 & Millipore & MAB3317 & Mouse & $1 / 1000$ & $1 / 200$ & Chicken anti-mouse \\
NF-kB (p65) & Abcam & AB7970 & Rabbit & $1 / 500$ & $1 / 50$ & Goat anti-rabbit \\
TIMP-3 & Millipore & MAB3318 & Mouse & $1 / 1000$ & $1 / 200$ & Chicken anti-mouse \\
\hline
\end{tabular}

2.3. Studies with Isolated Primary Human Macrophages. Peripheral blood mononuclear cells were isolated by differential centrifugation from whole blood of healthy donors, which were collected under South West 4 Research Ethics Committee reference 09/H0107/22. Blood (24 mL per donor) was diluted with Dulbecco's Phosphate Buffered Saline (PBS) without calcium and magnesium (Lonza) $1 \mathrm{x}$ (ratio 1:1). The diluted samples were subjected to density gradient separation on Ficoll Paque Plus (ratio 1:1) (GE Healthcare Life Sciences, Buckinghamshire, UK) and centrifuged. After centrifugation the PBMC layer was collected and washed in Hank's Balanced Salt Sodium (HBSS) with phenol red without calcium and magnesium (Lonza). Monocytes were isolated by adhering the peripheral blood mononuclear cells to tissue culture plastic for 2 hours at a concentration of $2.5 \times 10^{6}$ cells $/ \mathrm{mL}$. Monocytes were cultured in RPMI media with $2 \mathrm{mM}$ L-glutamine, $100 \mathrm{IU} / \mathrm{mL}$ penicillin, $100 \mu \mathrm{g} / \mathrm{mL}$ streptomycin, 10\% fetal bovine serum (FBS; Lonza, Sigma), and $20 \mathrm{ng} / \mathrm{mL}$ recombinant human macrophage-colonystimulating factor (R \& D systems). The medium included $85 \mu \mathrm{g} / \mathrm{mL}$ of fully oxidised low-density lipoprotein, oxLDL (Intracel) from day 5 when foam-cell macrophages (FCM) were generated. At Day 7, the medium was removed and replaced with RPMI media supplemented with 5\% FCS. Recombinant human interferon- $\gamma$ (10 ng/mL R \& D systems) and lipopolysaccharide-LPS $(200 \mathrm{ng} / \mathrm{mL}$, Escherichia coli 026:B6, Sigma-Aldrich) were added for 24 hours to generate classically activated macrophages (M1) [25] or recombinant human interleukin-4 (20 ng/mL R \& D systems) to obtain alternatively activated macrophages (M2) [26].

2.4. Reverse Transcription-Polymerase Chain Reaction (RT$P C R)$. RNA samples from macrophages were collected using RLT solution (Qiagen) with $\beta$-mercaptoethanol ( $\beta$-ME; $1: 100$ dilution). Total RNA was extracted using RNeasy Mini kit (Qiagen) according to the manufacturer's instructions. RNA was quantified using a Nanodrop Spectrophotometer and cDNA generated using a QuantiTect Reverse Transcription Kit (100-200 ng RNA per reaction Qiagen). Real-time quantitative PCR was performed in a Roche Light Cycler 1.5 to quantify the steady-state concentration of RNA using a QuantiTect SYBR Green PCR Kit and primers as detailed in Table 2. The reaction contained 3.6-7.3 ng RNA and $0.5 \mu \mathrm{M}$ primers. Denaturation for $15 \mathrm{~min}$ at $95^{\circ} \mathrm{C}$ was followed by 60 cycles of denaturation ( 15 seconds at $95^{\circ} \mathrm{C}$ ), annealing for 20 seconds, and extension for 25 seconds at $72^{\circ} \mathrm{C}$. Copy numbers of mRNA were calculated using standard curves constructed using the respective PCR products eluted from agarose gels. All fragments were sequenced to confirm identity (Cogenics, Takeley, UK). Primers used and their annealing temperatures are in Table 2.

2.5. Western Blotting. Adherent cells were washed with PBS on ice and then lysed with ice-cold $1 \%$ SDS lysis buffer. The Bicinchoninic acid (BCA) Kit (Pierce) was used to estimate protein concentration (all in duplicate) of cell lysates according to kit protocol. Protein concentration was read at $560 \mathrm{~nm}$ on a Multiskan Ascent (Thermo Electron Corporation) plate reader. NuPAGE Novex Bis-Tris Mini Gel system (Invitrogen, Life Technologies, Paisley, UK) was used for western blot experiments. $10 \mu \mathrm{g}$ of total protein was loaded on $4-12 \%$ Bis-Tris-HCl buffered ( $\mathrm{pH}$ 6.4) polyacrylamide precast gels (Invitrogen, Life Technologies, Paisley, UK) and run in MES-SDS Running Buffer 1x (Invitrogen, Life Technologies, Paisley, UK) according to manufacturer instructions. After electrophoresis, gels were transferred to a $0.45 \mu \mathrm{m}$ nitrocellulose membrane in NOVEX NuPAGE Transfer Buffer $1 \mathrm{x}$ plus $5 \%$ Methanol $(\mathrm{v} / \mathrm{v})$. Following transfer, the nitrocellulose membrane was blocked in 5\% milk solution in Tris buffered solution containing Tween (TBS-T) $(200 \mathrm{mM}$ Tris, $2 \%$ Tween 20, pH 7.6). The membrane was then incubated over night at $4^{\circ} \mathrm{C}$ using the antibodies described in Table 1 diluted in $2 \mathrm{~mL}$ of SignalBoost Solution 1 (CalbiochemNovabiochem Ltd., Nottingham, UK). The following day the membrane was incubated with $5 \%$ milk solution in TBS-T and then with the applicable horseradish peroxidase (HPR)labelled secondary antibody (Dako, Dorset, UK) diluted $1: 1000$ in SignalBoost Solution 2 (Calbiochem-Novabiochem Ltd., Nottingham, UK). After incubation the membrane was washed in TBS-T. To detect the peroxidise labelled proteins, an enhanced chemiluminescence (ECL) detection system (GE Healthcare Life Sciences, Buckinghamshire, UK) was used. The membrane was incubated with ECL reagent mix (solution A and solution B at a ratio of 1:1) and exposed to $\mathrm{X}$-ray film for the desired length of time. As a loading control $0.5 \mu \mathrm{g} / \mathrm{mL}$ of either anti- $\beta$-actin antibody (A2228, Sigma) or anti-GAPDH antibody (MAB374, Millipore) was used. Detected bands were quantified using a Bio-Rad GS-690 scanning densitometer (Bio-Rad, Hemel Hempstead, UK) and were normalised by relevant $\beta$-actin or GAPDH values. 
TABLE 2: Primers for quantitative RT-PCR.

\begin{tabular}{|c|c|c|c|c|}
\hline Primer & & Sequence & Annealing temp. $\left({ }^{\circ} \mathrm{C}\right)$ & Fragment size (bp) \\
\hline \multirow{2}{*}{ MMP-14 } & Forward & GGAGACAAGCATTGGGTGTT & \multirow{2}{*}{60} & \multirow{2}{*}{343} \\
\hline & Reverse & GGTAGCCCGGTTCTACCTTC & & \\
\hline \multirow{2}{*}{ TIMP-3 } & Forward & CTTCCGAGAGTCTCTGTGGCCTTA & \multirow{2}{*}{60} & \multirow{2}{*}{230} \\
\hline & Reverse & CTCGTTCTTGGAAGTCACAAAGCA & & \\
\hline \multirow{2}{*}{$\mathrm{COX}-2$} & Forward & GCCATGGGGTGGACTTAAATCATA & \multirow{2}{*}{60} & \multirow{2}{*}{168} \\
\hline & Reverse & CAGGGACTTGAGGAGGGTAGATCA & & \\
\hline \multirow{2}{*}{ CD206 } & Forward & CGGTGACCTCACAAGTATCCACAC & \multirow{2}{*}{58} & \multirow{2}{*}{216} \\
\hline & Reverse & TTCATCACCACACAATCCTCCTGT & & \\
\hline \multirow{2}{*}{$\mathrm{IkB} \alpha$} & Forward & CTACTGGACGACCGCCACGACAGC & \multirow{2}{*}{60} & \multirow{2}{*}{58} \\
\hline & Reverse & CGAGGCGGATCTCCTGCAGCTCCTTG & & \\
\hline \multirow{2}{*}{$36 \mathrm{~B} 4$} & Forward & GCCAGCGAAGCCACGCTGCTGAAC & \multirow{2}{*}{60} & \multirow{2}{*}{76} \\
\hline & Reverse & CGAACACCTGCTGGATGACCAGCCC & & \\
\hline
\end{tabular}

2.6. Statistical Methods. For histomorphometry, differences between categorical and continuous variables were analysed by using an independent Student's $t$-test in case of normally distributed data, and a Mann-Whitney $U$ test in case of not normally distributed data. Differences between ordinal and continuous data were analysed by using a Kruskal-Wallis test, in case of not normally distributed data. Differences between dichotomous and categorical data were analysed by using a Chi-square test. Differences between 2 continuous variables were analysed with a Spearman correlation test in case of not normally distributed data. In all cases $P<$ 0.05 was considered significant. All statistical analysis was performed with SPSS version 17 (SPSS Inc., Chicago, IL, USA). Quantitative RT-PCR results normalised to the values for $18 \mathrm{~s}$ RNA were transformed to logarithms and analysed by ANOVA followed by Student's $t$-test with Bonferroni correction.

\section{Results}

3.1. MMP-14 and TIMP-3 mRNA Expression in Human Primary Macrophages and FCMs In Vitro. Human monocytederived macrophages were differentiated with M-CSF (MMs) and some from each batch were treated with oxidised LDL to generate foam-cell macrophages (FCMs), as previously validated in our laboratory [27]. Conversion of MMs to FCMs did not affect mRNA expression of MMP-14 or TIMP3 (Figure $1(\mathrm{a})$ ) or COX -2 and $\mathrm{I} \kappa \mathrm{B} \alpha$, markers of proinflammatory activation, or CD206, a gene that is present constitutively but upregulated by anti-inflammatory activation [12, 13] (Figure 1(a)). Proinflammatory activation with LPS and IFN $\gamma$ (referred to as M1 conditions [26]) significantly increased expression of MMP-14 by 4.5 - and 7.3-fold, I $\kappa \mathrm{B} \alpha$ mRNA (a surrogate marker of $\mathrm{NF} \kappa \mathrm{B}$ signalling [28]) by 7.5- and 6.7fold, and COX-2 mRNA by 146- and 187-fold, in macrophages (MM1) and FCMs (FCM1), respectively (Figure 1(a)). By contrast, proinflammatory activation decreased mRNA levels of TIMP- 3 by $74 \%$ and $89 \%$ and CD206 by $81 \%$ and $86 \%$ in macrophages or FCMs, respectively (Figure 1(a)). Antiinflammatory activation with IL-4 (referred to as M2 conditions [26]) had no effect on MMP-14, COX- 2 or I $\kappa \mathrm{B} \alpha$ mRNA levels but significantly increased TIMP-3 mRNA expression by 1.9- and 2.6-fold and CD206 expression by 7.5- and 9.9-fold in MMs (MM2) and FCMs (FCM2), respectively (Figure 1(a)).

3.2. MMP-14 and TIMP-3 Protein Expression in Macrophages and FCMs In Vitro. Consistent with the mRNA data, proinflammatory activation significantly elevated protein levels of MMP-14 and COX-2 and significantly decreased those of TIMP-3 and CD206 in nonfoamy macrophages (Figures 1(b) and 1(c)). Anti-inflammatory activation (MM2) also increased macrophage TIMP-3 and CD206 protein levels (Figures $1(\mathrm{~b})$ and $1(\mathrm{~d})$ ), consistent with the mRNA data. We conclude that proinflammatory macrophages are MMP- $14^{\text {hi }}$ TIMP- $3^{\text {lo }}$ whereas unactivated and antiinflammatory stimulated macrophages are comparatively MMP-14 ${ }^{\text {lo }}$ TIMP- $3^{\text {hi }}$. Foam-cell formation significantly increased protein expression of MMP-14 and decreased TIMP-3 levels (Figures 1(e) and 1(f)), despite not changing the mRNA levels, in agreement with our previous findings in during rabbit macrophage foam-cell formation [7]. These results could be explained by the additional involvement of a posttranscriptional mechanism, such as regulation by microRNA (miR). Indeed, we recently showed that high levels of miR-24 in nonfoamy macrophages limit macrophage MMP-14 protein expression [29]. We observed a 70\% decrease in miR-24 levels in FCMs compared to MMs (Figure $1(\mathrm{~g})$ ), consistent with the increased MMP-14 protein levels. Interestingly, in contrast to mRNA expression, pro- or anti-inflammatory activation of FCMs did not affect MMP-14 protein expression (Figure $1(\mathrm{~h})$ ), which suggests that the effect of the miR is dominant. Pro- or anti-inflammatory activation of FCMs did not affect TIMP-3 protein expression either when compared to nonactivated FCMs (Figure 1(i)). TIMP-3 protein expression may also be subject to $\mathrm{miR}$ regulation but this has not yet been demonstrated in macrophages. In summary, our results imply that nonfoamy macrophages are MMP- $14^{\text {hi }}$ TIMP- $3^{\text {lo }}$ when classically activated whereas FCMs are MMP- $14^{\text {hi }}$ TIMP-3 ${ }^{\text {lo }}$ at the protein level irrespective of their activation status. 


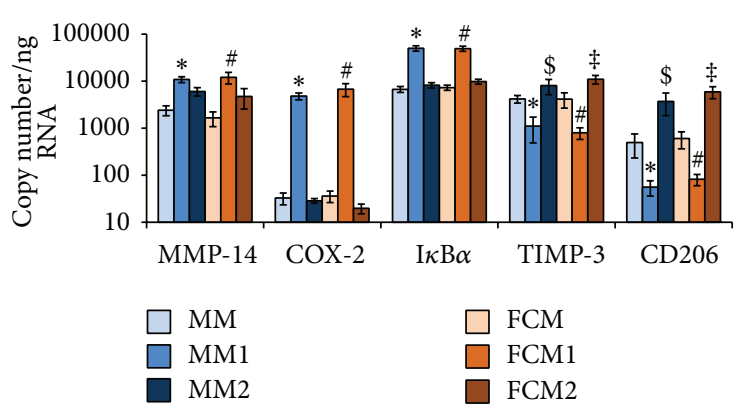

(a)

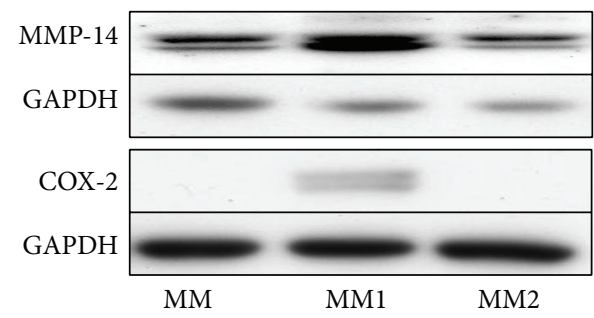

(c)

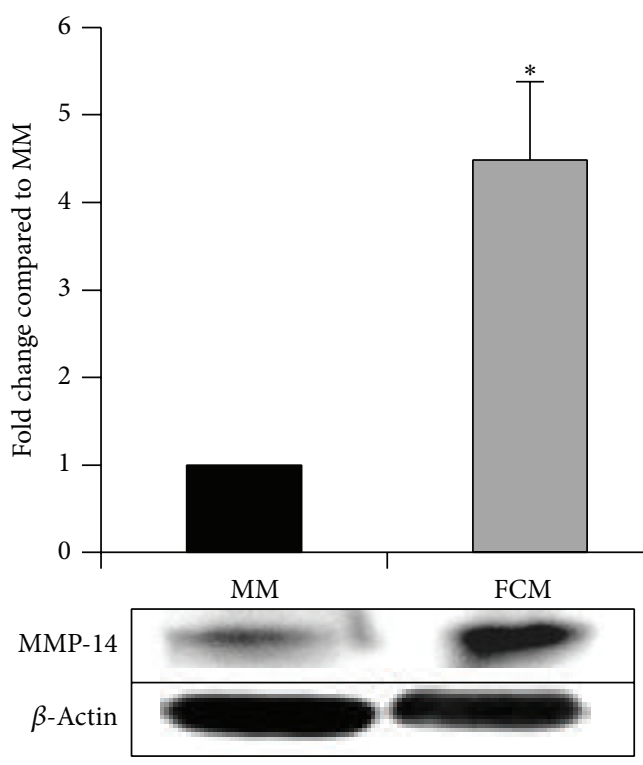

(e)

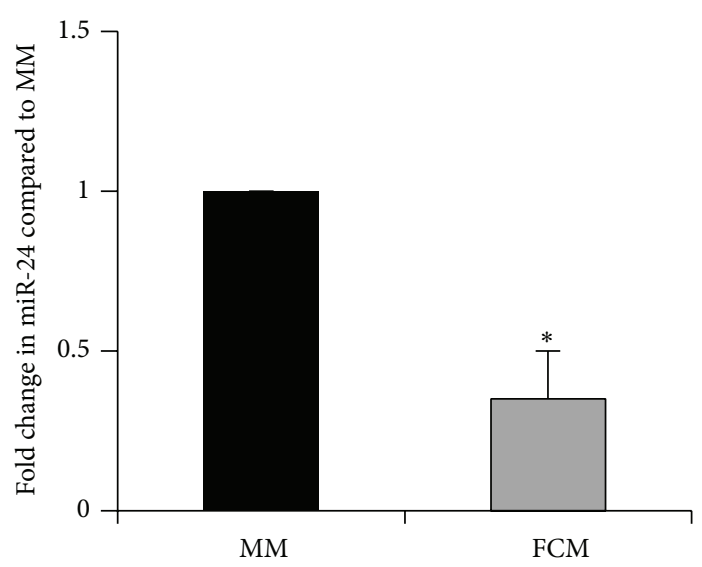

(g)

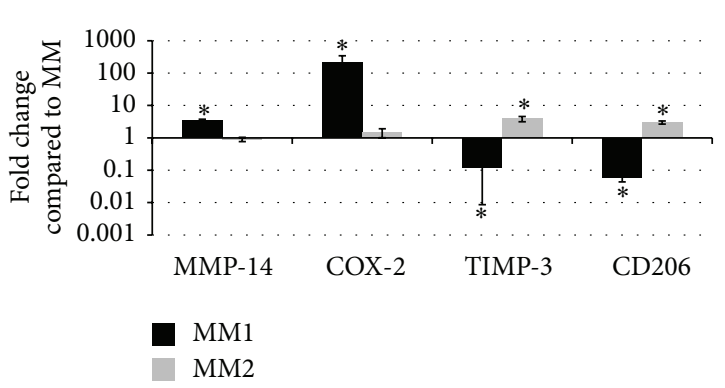

(b)

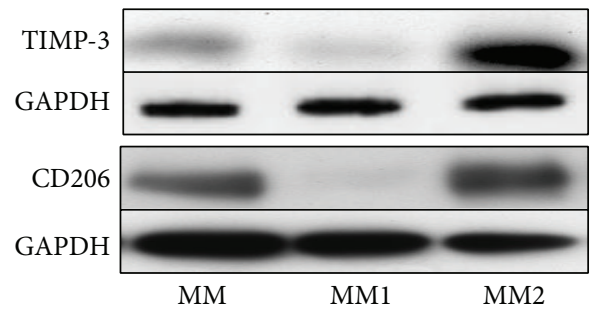

(d)

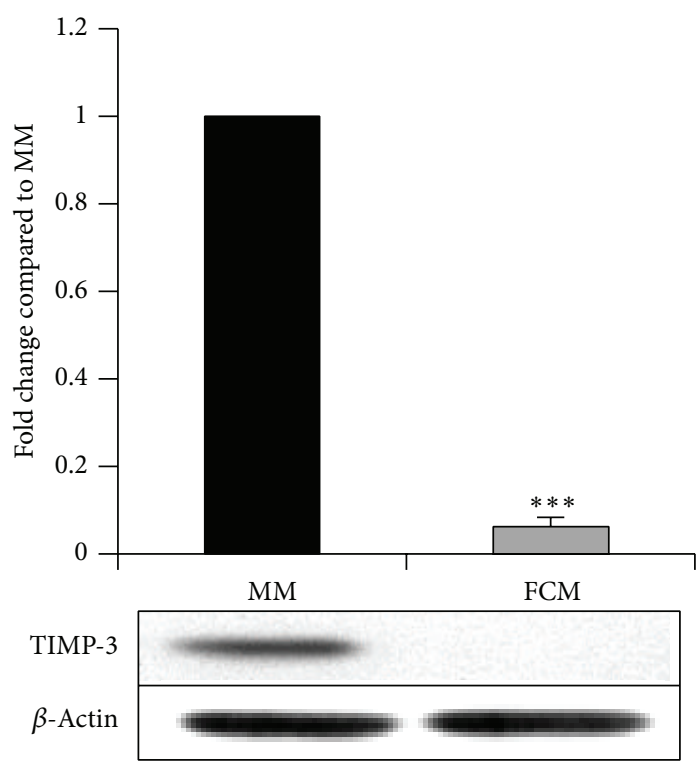

(f)

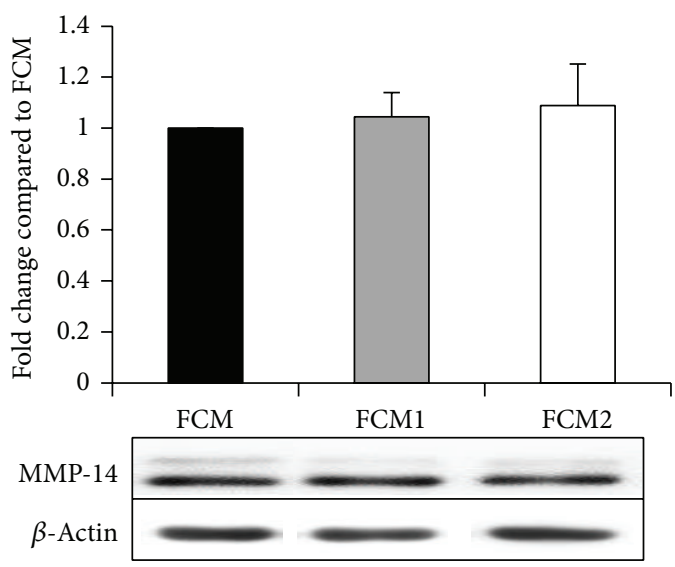

(h)

FIgUre 1: Continued. 


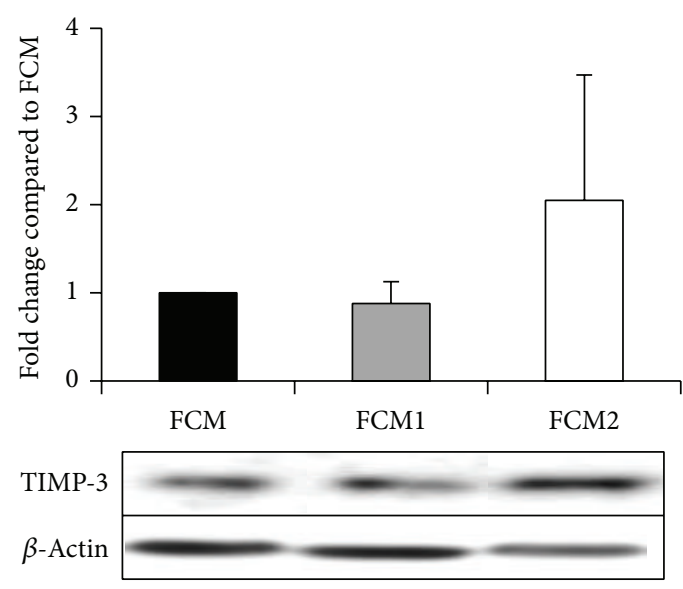

(i)

FIGURE 1: (a) mRNA expression of M-CSF differentiated macrophages (MM) and foam-cell macrophages (FCM) after activation with IFN $\gamma$ and LPS (MM1 and FCM1) or IL-4 (MM2 and FCM2). Data are mean \pm SEM; $n=7 ; *=P<0.05$ compared to MM and MM2; \# = $P<0.05$ compared to FCM and FCM2; $\$=P<0.05$ compared to MM and MM1; and $\neq=P<0.05$ compared to FCM and FCM1. ((b)-(d)) Densitometric quantification and representative Western blots for MMP-14, COX-2, TIMP-3, and CD206 in M-CSF differentiated macrophages (MM) or after activation with IFN $\gamma$ and LPS (MM1) or IL-4 (MM2). Data are mean \pm SEM, ${ }^{*} P<0.05$ compared to MM, $n=8$. ((e)-(f)) Densitometric quantification and representative Western blots for MMP-14 (e) and TIMP-3 (f) in M-CSF differentiated macrophages $(\mathrm{MM})$ and foam-cell macrophages (FCM). Data are mean $\pm \mathrm{SEM},{ }^{*} P<0.05$ compared to $\mathrm{MM},{ }^{*} P<0.001$ compared to MM, $n=4$. (g) QPCR expression of miR-24 in M-CSF differentiated macrophages (MM) and foam-cell macrophages (FCM). Data are mean \pm SEM, ${ }^{*} P<0.05$ compared to MM. ((h)-(i)) Densitometric quantification and representative Western blots for MMP-14 (h) and TIMP-3 (i) in foam-cell macrophages (FCM) after activation with IFN $\gamma$ and LPS (FCM1) or IL-4 (FCM2). Data are mean \pm SEM; $n=4$.

3.3. Localisation of MMP-14 and TIMP-3 in Human Carotid Atherosclerotic Plaques. Macrophages and FCMs were identified in plaques using immunohistochemistry for CD68. The CD68 positive cells varied widely in morphology from large, highly foamy cells to those with very few or no lipid inclusions. We counted all cells irrespective of morphology. MMP-14 positive macrophages/FCMs were found predominantly in the shoulder regions (SR) of atheromatous carotid plaques (Figures 2(a)-2(c)), whereas TIMP-3 positive macrophages/FCMs occurred predominantly within and around the fibrous cap (FC) of fibrous atheromatous plaques (Figures 2(d)-2(e)). The specificity of the antibodies used is demonstrated in Figure 2. In line with our previous findings [7], regions of plaques tended to be either MMP-14 positive and TIMP-3 negative (Figure 2(g) compared to Figure 2(h)) or MMP-14 negative and TIMP-3 positive (Figure 2(i) compared to Figure 2(j)). Dual Immunohistochemistry (as described previously [24]) revealed that in plaque regions with abundant CD68 positive FCMs, MMP-14 colocalised with nuclear-localised NF- $\kappa$ B p65 subunit, a recognised marker of proinflammatory activation. When counted, approximately $80 \%$ of MMP-14 positive FCMs also had nuclear-localised NFאB (Figures 3(a)-3(d)), which implies that proinflammatory activation associated with MMP-14 upregulation in macrophages/FCMs in plaques. To gain further insight, we employed arginase-1, which is a marker for anti-inflammatory mouse macrophages [30] and is downregulated in a subpopulation of human FCMs in advanced plaques [31]. Arginase-1 and MMP-14 were coexpressed in more than $50 \%$ of intraplaque macrophages/FCMs and the number of arginase- 1 and MMP-14 positive macrophages was correlated in advanced plaques $\left(r^{2}=0.960 ; P<0.0001\right.$, Figure 4). These results are consistent with our in vitro data showing high MMP-14 protein levels in FCMs irrespective of M1/M2 activation state (Figure 4). Conversely to MMP14 , less than $1 \%$ of TIMP-3 positive macrophages/FCMs demonstrated nuclear-localised NF- $\kappa$ B p65 (Figure 5), implying that few were classically activated. Approximately $80 \%$ of macrophages/FCMs that were TIMP-3 positive also stained for CD206, which we showed to be expressed in unactivated (MM) and alternatively activated (MM2) macrophages (Figures 3(f)-3(i)). We therefore concluded that CD206 and TIMP-3 positive macrophages/FCMs in plaques could be unactivated or IL- 4 activated.

3.4. Relationship of MMP-14 and TIMP-3 Staining to Histological Features of Plaque Stability and Occurrence of Symptoms in Carotid Atherosclerotic Plaques. We next investigated the association between MMP-14 and TIMP-3 staining and plaque composition. Carotid plaques entered into AtheroExpress are given an initial classification into atheromatous, fibrous-atheromatous, or fibrous plaques based on a large lipid core, high abundance of macrophages, and low abundance of smooth muscle cells (SMCs) and collagen content $[20,21,32]$ (Table 3). Of note, male and older patients are overrepresented in the group with atheromatous plaques (Table 4). By comparison with these previous assignments we found that the percentage of MMP-14 (Figure 6(a)) and COX-2 (Figure 6(b)) positive macrophages/FCMs was significantly greater in atheromatous or fibrous-atheromatous 


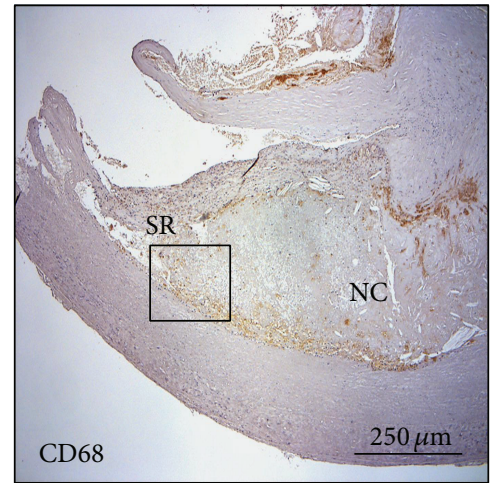

(a)

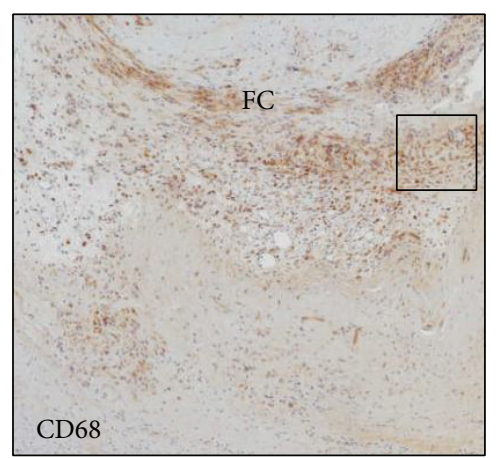

(d)

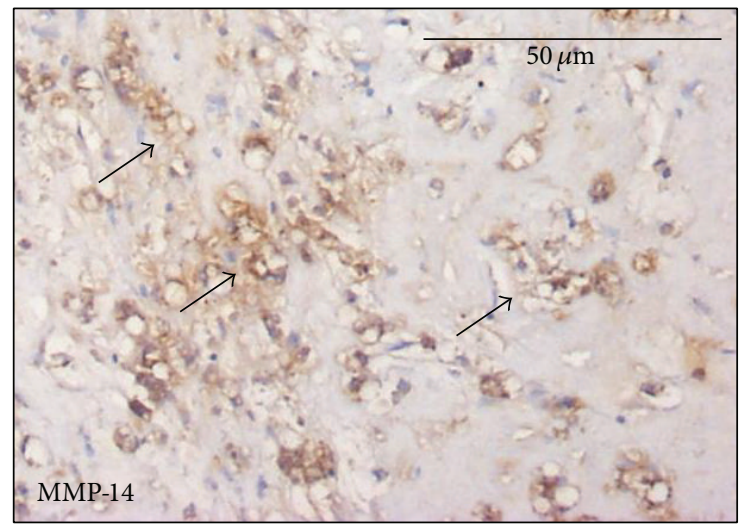

(g)

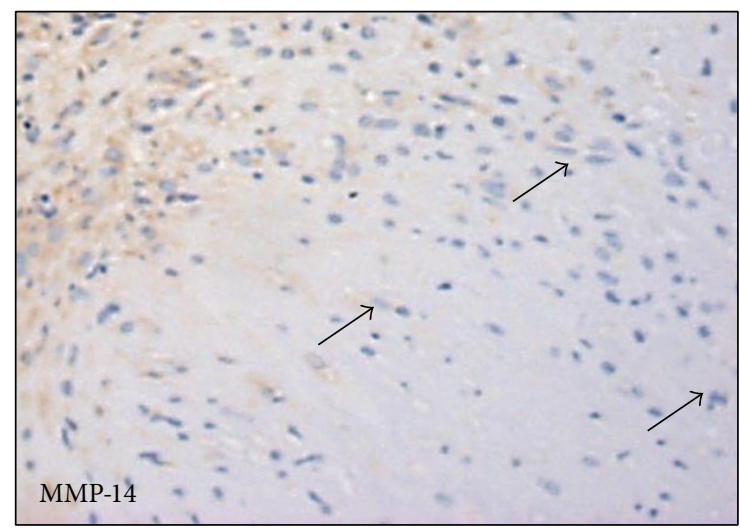

(i)

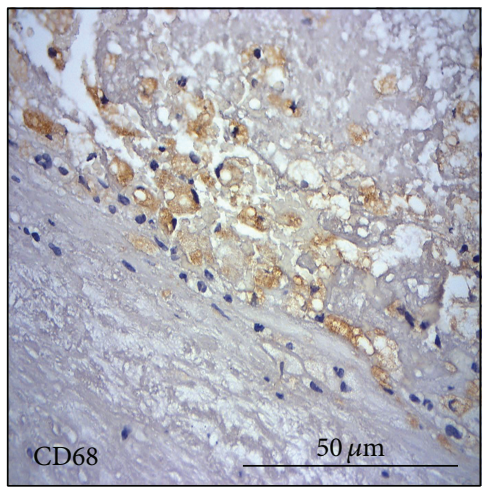

(b)

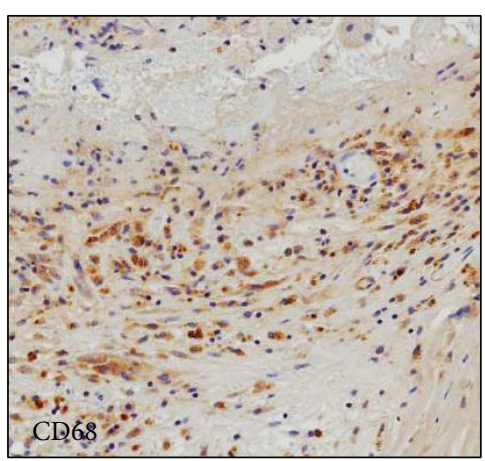

(e)

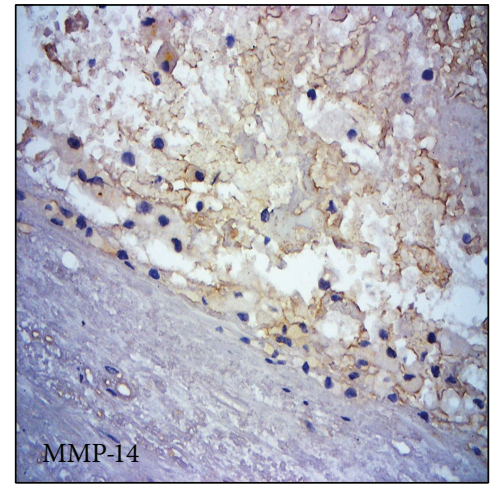

(c)

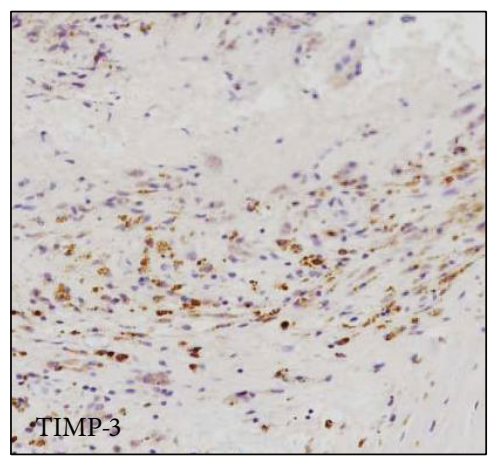

(f)

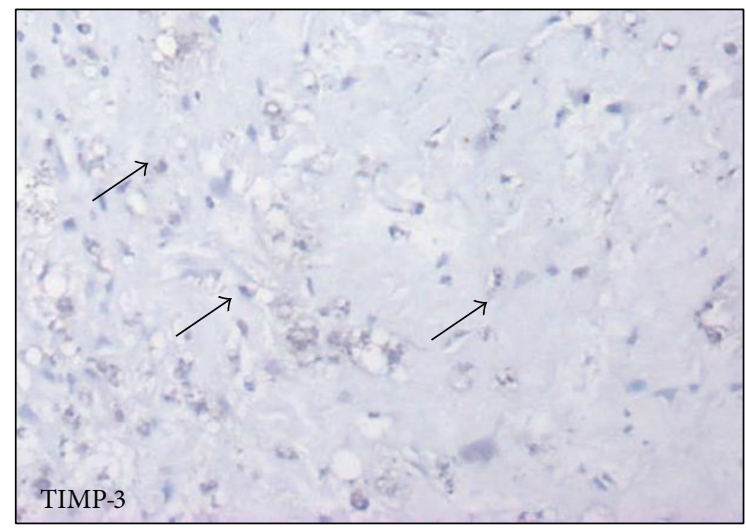

(h)

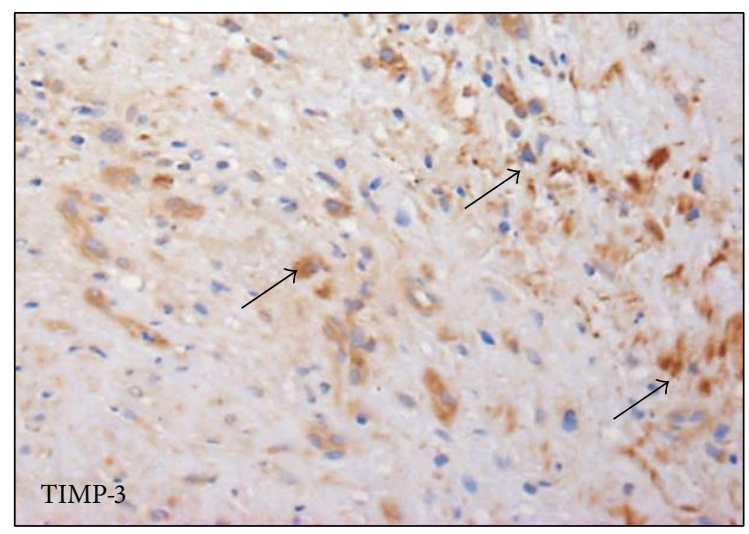

(j)

FIGURE 2: Continued. 


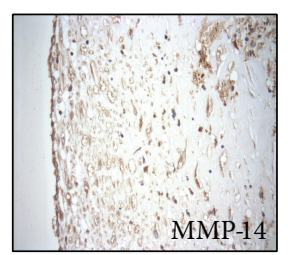

(k)

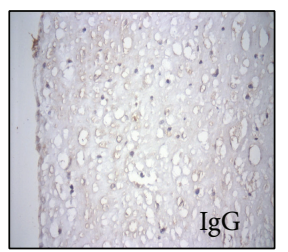

(p)

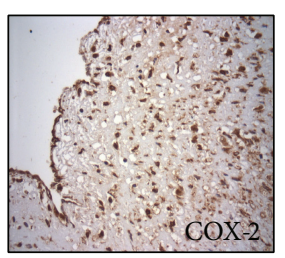

(l)

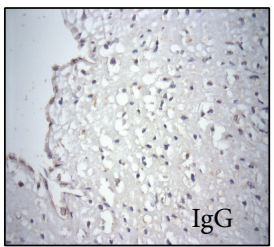

(q)

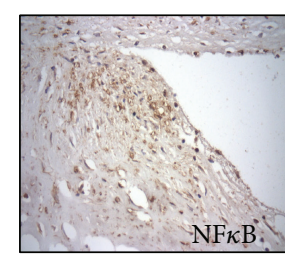

(m)

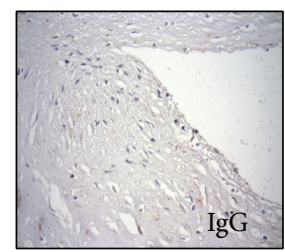

$(\mathrm{r})$

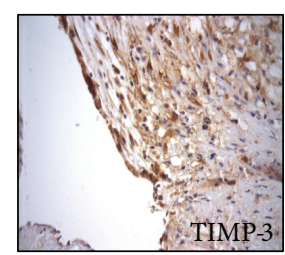

(n)

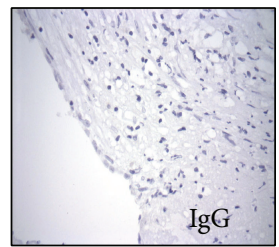

(s)

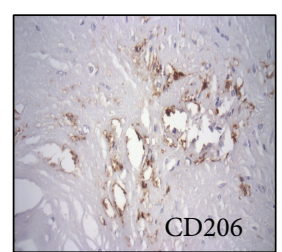

(o)

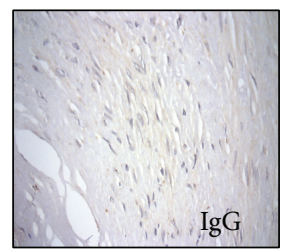

$(\mathrm{t})$

Figure 2: ((a)-(f)) Immunolocalisation of macrophages (CD68), MMP-14, and TIMP-3 in human atheromatous ((a)-(c)) and fibrousatheromatous ((d)-(e)) carotid plaques. Panels (b)-(c) and (e)-(f) are higher magnification fields of boxes depicted in panels (a) and (d), respectively. SR denotes shoulder region, NC denotes necrotic core, and FC denotes fibrous cap. ((g)-(j)) Immunohistochemistry of atheromatous carotid plaques demonstrating MMP- $14^{\text {hi }}$ TIMP- $3^{\text {lo }}((\mathrm{g})$ and $(\mathrm{h})$, serial sections $)$ and MMP- $14^{\text {lo }}$ TIMP- $3^{\text {hi }}((\mathrm{i})$ and $(\mathrm{j})$, serial sections) foam-cell macrophage regions. Arrows indicate same cells in adjoining serial sections. $((\mathrm{k})-(\mathrm{t}))$ Immunohistochemical staining (brown product colour) of carotid plaque sections for (k) MMP-14, (l) COX-2, (m) NFkB, (n) TIMP-3, and (o) CD206 and their relevant control IgG on serial sections ((p)-(t), resp.).

compared to fibrous plaques. By contrast, the percentage TIMP-3 (Figure 6(a)) and CD206 (Figure 6(b)) positive macrophages/FCMs increased in fibrous compared to fibrous-atheromatous and atheromatous carotid plaques. Despite the small number of asymptomatic patients in our cohort, significantly higher MMP-14 positivity was found in plaques from symptomatic compared to asymptomatic patients (Figure 6(c)). There was also a trend towards lower TIMP-3 positivity in plaques from symptomatic patients (Figure 6(c)).

Amplifying the relationships to overall plaque designation, we found a higher percentage of MMP-14 positive macrophages/FCMs was associated with plaques showing high lipid and macrophage content or decreased SMC number, which are related to plaque instability, whereas the converse was observed for TIMP-3 positive FCMs (Figure 7). TIMP-3 positive macrophages/FCMs were also significantly associated with increased collagen content and reduced plaque neovascularisation (Figure 7), other markers of plaque stability. Furthermore, the percentage of MMP-14 positive macrophages/FCMs across all the sections correlated strongly with total macrophage number and negatively with SMC number (Table 5). Percentage positivity for MMP-14 and COX-2 also correlated with each other (Table 5). Conversely, the percentage of TIMP-3 positive macrophages/FCMs strongly correlated negatively with macrophage number and positively with SMC number (Table 5). Positivity for TIMP-3 correlated directly with CD206 and inversely with MMP-14 (Table 5). Other characteristics including the presence of calcification, intraplaque haemorrhage, and thrombus formation were also recorded. Positivity of MMP-14 or TIMP-3 was unrelated to plaque calcification (data not shown). However, plaques with intraplaque haemorrhage had a significantly higher percentage of MMP-14 positive macrophages/FCMs and tended to have a lower percentage of TIMP-3 positive FCMs (Table 6).

\section{Discussion}

Our main new findings are that macrophages activated with proinflammatory stimuli or foam-cell macrophages (FCMs) irrespective of inflammatory activation are MMP$14^{\text {hi }}$ TIMP- $3^{\text {lo }}$ compared to unstimulated (M-CSF differentiated; MM) or anti-inflammatory (IL-4 activated; MM2) macrophages. Furthermore, MMP-14 positive FCMs are more abundant and TIMP-3 positive FCMs are less abundant in plaques with vulnerable rather than stable characteristics.

Multiple macrophage and FCM phenotypes have been previously described $[33,34]$. Classical macrophage activation in vitro with proinflammatory stimuli is characterised by NF- $\kappa$ B-dependent upregulation of a variety of additional proinflammatory mediators and enzyme systems, including COX-2. By contrast, in vitro activation with antiinflammatory stimuli such as IL-4 or IL-13 mediates a distinct transcriptomic response dependent on STAT-6 phosphorylation $[26,33]$. Our results demonstrate a prominent effect of classical activation on MMP-14 mRNA expression in macrophages and FCM [35], consistent with previous work on human monocytes [36] and macrophages [35] demonstrating that NF- $\kappa \mathrm{B}$ activation facilitates MMP-14 expression. Formation of foam cells per se did not lead to pro- or antiinflammatory macrophage activation (Figure 1), consistent with previous studies $[16,37]$. However, transformation to FCM increased MMP-14 protein expression, regardless of proinflammatory activation. FCMs appear resistant to M1 


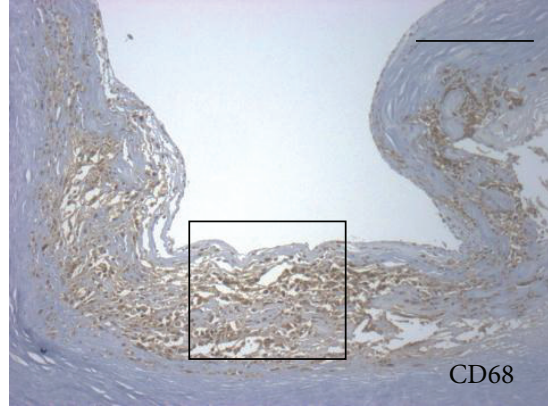

(a)

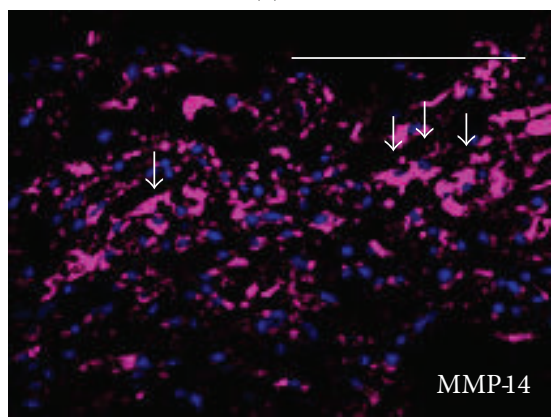

(b)

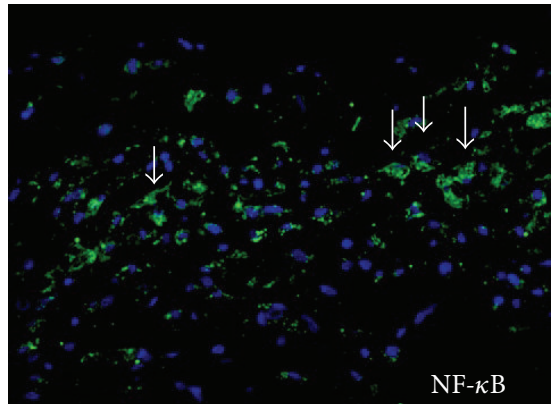

(c)

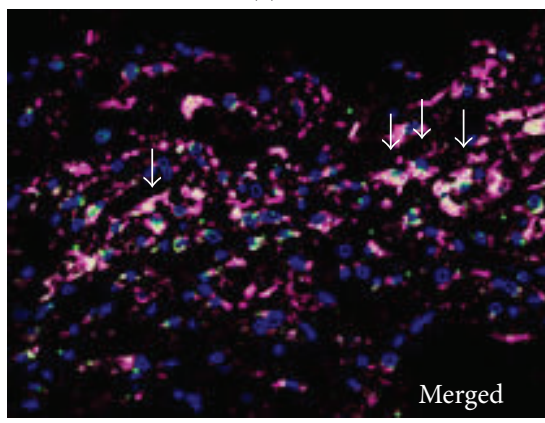

(d)

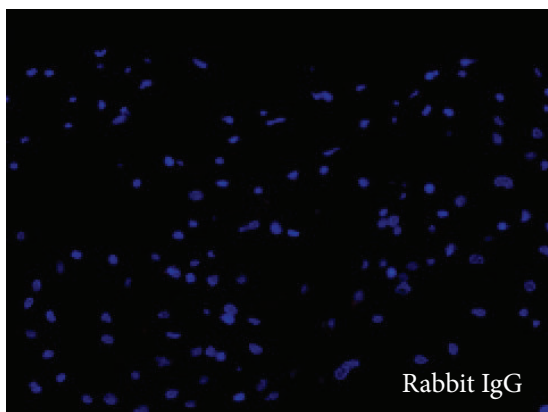

(e)

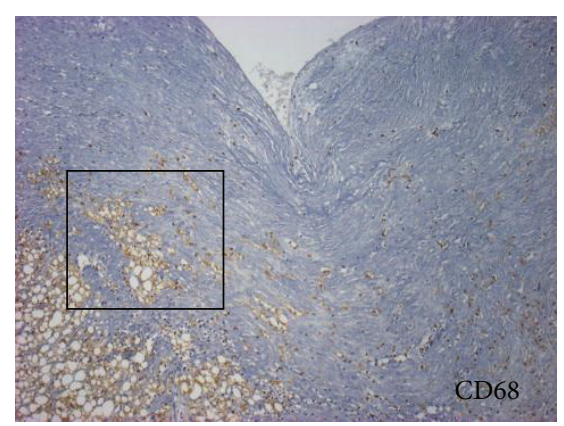

(f)

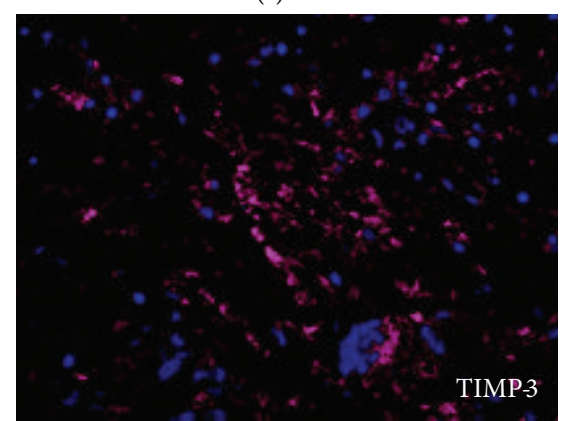

(g)

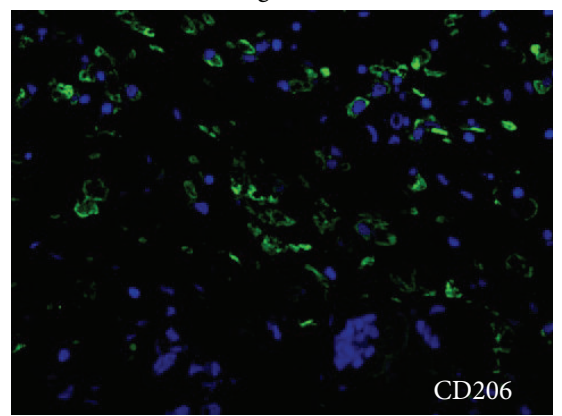

(h)

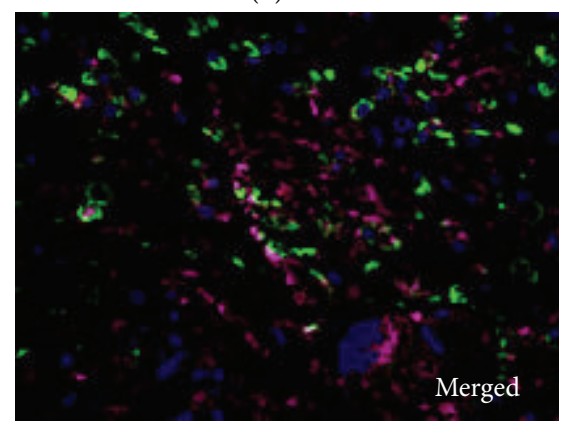

(i)

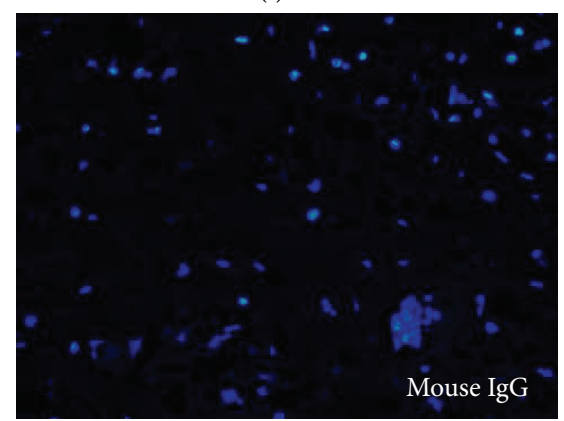

(j)

Figure 3: Continued. 


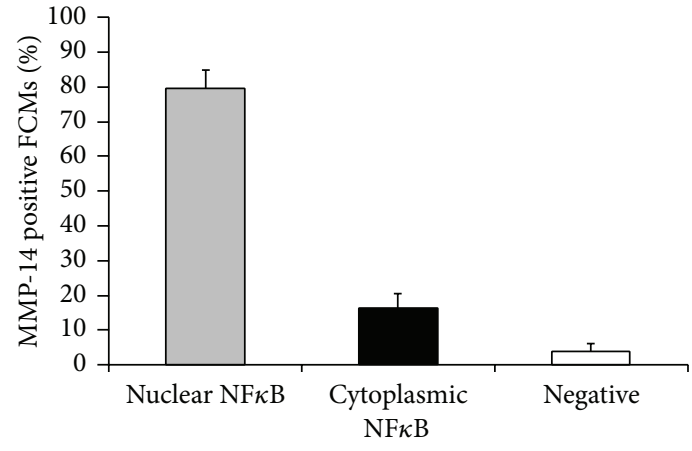

$(\mathrm{k})$

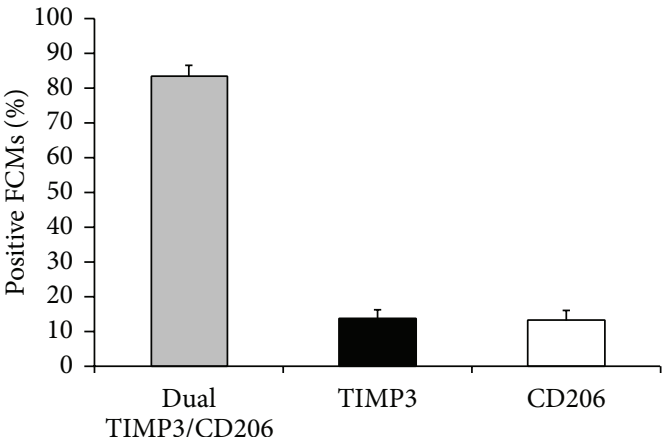

(l)

FIGURE 3: (a)-(j) Representative images of immunohistochemical labelling of unstable ((a)-(e)) and stable ((f)-(j)) human carotid artery atherosclerotic plaques, for macrophages (CD68, panels (a) and (f)), and colocalisation of nuclear-localised NFkB with MMP-14 ((b)-(d)), and CD206 with TIMP-3 ((h)-(j)), with nuclei counterstained with DAPI (blue). Box in panels (a) and (f) represents higher magnification in panels (b)-(e) and (g)-(j), respectively. Scale bar in (a) represents $250 \mu \mathrm{m}$ and is applicable to panels (a) and (f), whereas scale bar in (b) represents $100 \mu \mathrm{m}$ and is applicable to panels (b)-(e) and (g)-(j). Arrows in panels (b)-(d) indicate MMP-14 +ve FCMs with nuclear NF $\kappa$ B. Panels (e) and (j) represent negative controls where the primary antibodies were replaced with the relevant species IgG. (k) The percentage of MMP-14 positive FCMs that also stained for nuclear-localised NFא B was significantly greater $\left({ }^{* * *} P<0.001\right)$ compared to only cytoplasmic $\mathrm{NF} \kappa \mathrm{B}$ or no staining. (1) The percentage of TIMP-3 positive FCMs that stained also for CD206 was significantly greater $\left({ }^{* * *} P<0.001\right)$ compared to those stained for TIMP-3 or CD206 alone.

TABLE 3: Atherosclerotic plaque characteristics.

\begin{tabular}{|c|c|c|c|c|c|c|}
\hline \multirow{4}{*}{$\begin{array}{l}\text { Plaque characteristics } \\
\% \text { fat/atheroma } \\
\text { No/ }<40 \% />40 \%\end{array}$} & \multirow{2}{*}{$\begin{array}{l}\text { Plaque phenotype } \\
\text { Fibrous }\end{array}$} & \multicolumn{4}{|c|}{ Gradation } & \multirow{4}{*}{$\begin{array}{l}P \text { value } \\
0.0001\end{array}$} \\
\hline & & \multicolumn{2}{|c|}{$15(50 \%)$} & $15(50 \%)$ & $0(0 \%)$ & \\
\hline & Fibrous-atheromatous & \multicolumn{2}{|c|}{$0(0 \%)$} & $11(92 \%)$ & $1(8 \%)$ & \\
\hline & Atheromatous & \multicolumn{2}{|c|}{$0(0 \%)$} & $2(5 \%)$ & $35(95 \%)$ & \\
\hline \multirow{3}{*}{$\begin{array}{l}\text { Collagen semiquantitative } \\
\text { (minor/moderate/heavy) }\end{array}$} & Fibrous & \multicolumn{2}{|c|}{$1(4 \%)$} & $14(45 \%)$ & $15(52 \%)$ & \multirow{6}{*}{0.0001} \\
\hline & Fibrous-atheromatous & \multicolumn{2}{|c|}{$2(17 \%)$} & $7(58 \%)$ & $3(25 \%)$ & \\
\hline & Atheromatous & \multicolumn{2}{|c|}{$17(46 \%)$} & $19(51 \%)$ & $1(3 \%)$ & \\
\hline \multirow{3}{*}{$\begin{array}{l}\text { SMC semiquantitative } \\
\text { (no/minor/moderate/heavy) }\end{array}$} & Fibrous & $0(0 \%)$ & $0(0 \%)$ & $10(33 \%)$ & $20(67 \%)$ & \\
\hline & Fibrous-atheromatous & $0(0 \%)$ & $3(25 \%)$ & $7(58 \%)$ & $2(17 \%)$ & \\
\hline & Atheromatous & $2(5 \%)$ & $30(81 \%)$ & $5(14 \%)$ & $0(0 \%)$ & \\
\hline \multirow{3}{*}{$\begin{array}{l}\text { SMC quantitative } \\
\% \text { plaque area median }[\mathrm{IQR}]\end{array}$} & Fibrous & \multicolumn{4}{|c|}{$4.31[1.73-5.69]$} & \multirow{3}{*}{0.0001} \\
\hline & Fibrous-atheromatous & & 1.31[ & $4.62]$ & & \\
\hline & Atheromatous & & 0.53 & $1.07]$ & & \\
\hline \multirow{3}{*}{$\begin{array}{l}\text { Macrophage } \\
\text { semiquantitative } \\
\text { (no/minor/moderate/heavy) }\end{array}$} & Fibrous & $7(23 \%)$ & $20(67 \%)$ & $3(10 \%)$ & $0(0 \%)$ & \multirow{3}{*}{0.0001} \\
\hline & Fibrous-atheromatous & $3(25 \%)$ & $6(50 \%)$ & $2(17 \%)$ & $1(8 \%)$ & \\
\hline & Atheromatous & $0(0 \%)$ & $0(0 \%)$ & $19(51 \%)$ & $18(49 \%)$ & \\
\hline \multirow{3}{*}{$\begin{array}{l}\text { Macrophage quantitative } \\
\% \text { plaque area median [IQR] }\end{array}$} & Fibrous & \multicolumn{4}{|c|}{$0.20[0.05-0.63]$} & \multirow{3}{*}{0.0001} \\
\hline & Fibrous-atheromatous & & 0.15[ & $0.50]$ & & \\
\hline & Atheromatous & & 1.13[ & $2.52]$ & & \\
\hline \multirow{3}{*}{$\begin{array}{l}\text { Thrombus present } \\
\text { (no/minor/moderate/heavy) }\end{array}$} & Fibrous & $13(43 \%)$ & $14(47 \%)$ & $2(7 \%)$ & $1(3 \%)$ & \multirow{3}{*}{0.0001} \\
\hline & Fibrous-atheromatous & $2(17 \%)$ & $7(58 \%)$ & $1(8 \%)$ & $2(17 \%)$ & \\
\hline & Atheromatous & $4(11 \%)$ & $10(27 \%)$ & $16(43 \%)$ & $7(19 \%)$ & \\
\hline \multirow{3}{*}{$\begin{array}{l}\text { CD34 staining } \\
\text { Number of vessels median } \\
{[\text { IQR] }}\end{array}$} & Fibrous & \multicolumn{4}{|c|}{6.7 [3.8-9.5] } & \multirow{3}{*}{0.5000} \\
\hline & Fibrous-atheromatous & \multicolumn{4}{|c|}{$6.5[3.8-10.4]$} & \\
\hline & Atheromatous & \multicolumn{4}{|c|}{$7.0[4.3-11.0]$} & \\
\hline
\end{tabular}

$P$ values for differences among the three groups of semiquantitative histological markers and percentage of macrophages and smooth muscle cells were determined using the Kruskal-Wallis test. 


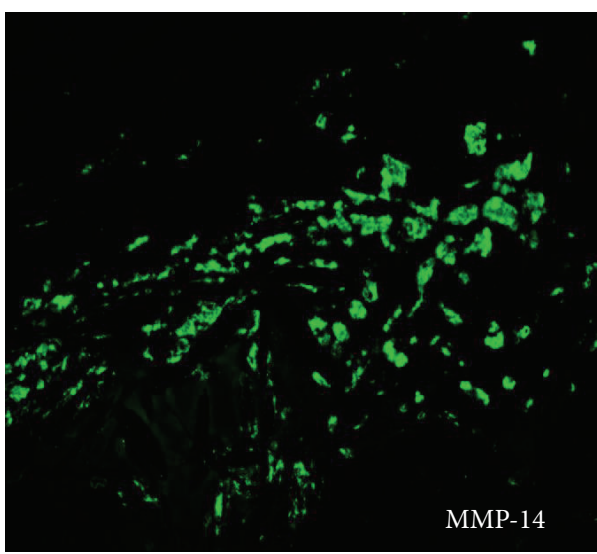

(a)

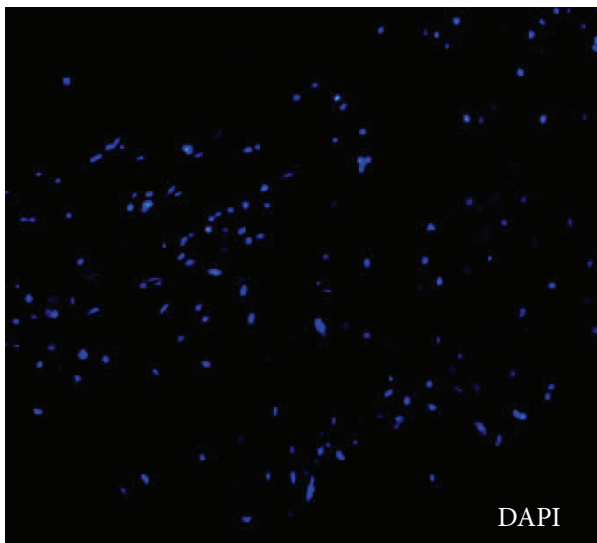

(c)

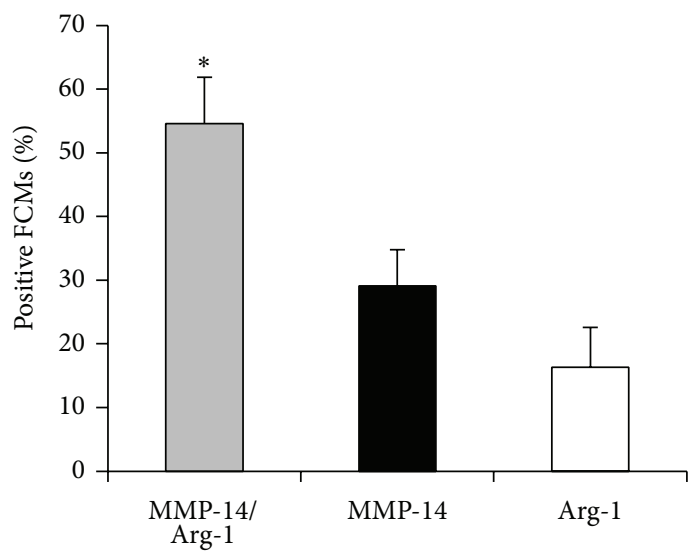

(e)

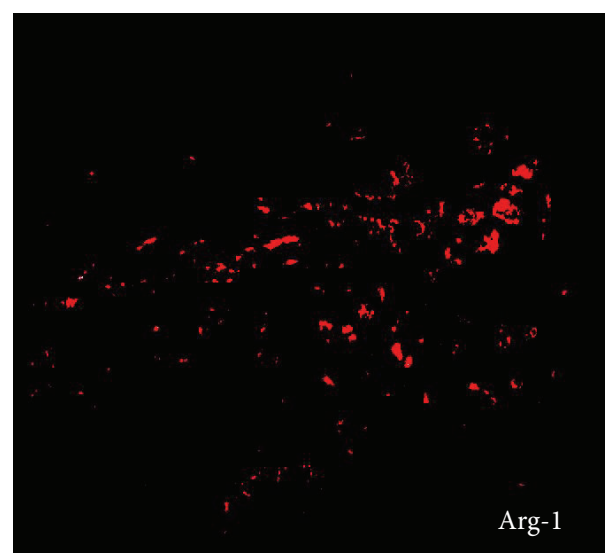

(b)

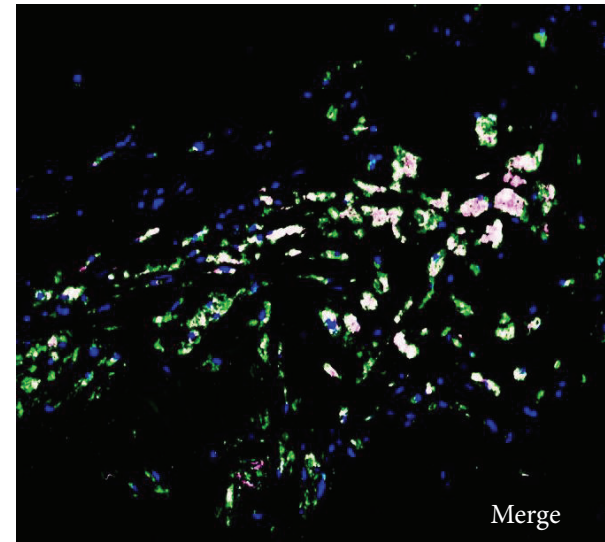

(d)

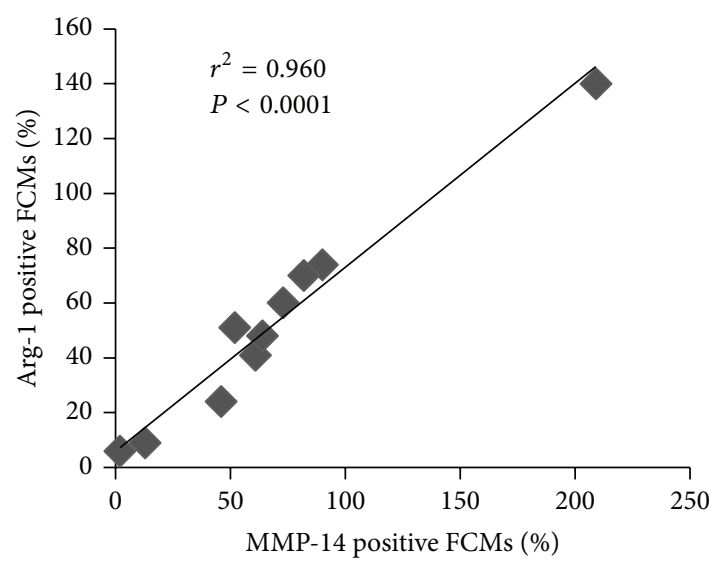

(f)

FIGURE 4: ((a)-(d)) Representative images of immunohistochemical labelling of advanced human carotid artery atherosclerotic plaques, for colocalisation of MMP-14 (a) with arginase-1 (b), with nuclei counterstained with DAPI (blue; (c)). Scale bar in (a) represents $100 \mu \mathrm{m}$ and is applicable to panels (a)-(d). (e) The percentage of MMP-14 positive FCMs that also stained for arginase-1 was significantly greater compared to only MMP-14 or arginase- $1\left({ }^{*} P<0.05 ; n=10\right.$ per group; data expressed as mean \pm SEM). (f) Correlation of arginase-1 and MMP-14 positive macrophages in human carotid artery atherosclerotic plaques $\left(r^{2}=0.960 ; P<0.0001 ; n=10\right)$.

and M2 cytokine-stimulation but employ a posttranscriptional pathway to modulate MMP-14 protein expression and subsequent activity. Indeed we have recently identified the involvement of a microRNA, miR-24, in the direct regulation of macrophage MMP-14 protein expression [29]. Further evidence for a novel pathway in regulating FCM MMP14 protein expression is provided by our demonstration that MMP-14 positive macrophages/FCMs in plaques correlated with classical activation markers, COX-2 and nuclearlocalised $\mathrm{NF}-\kappa \mathrm{B}$, and with the well-recognised marker of 


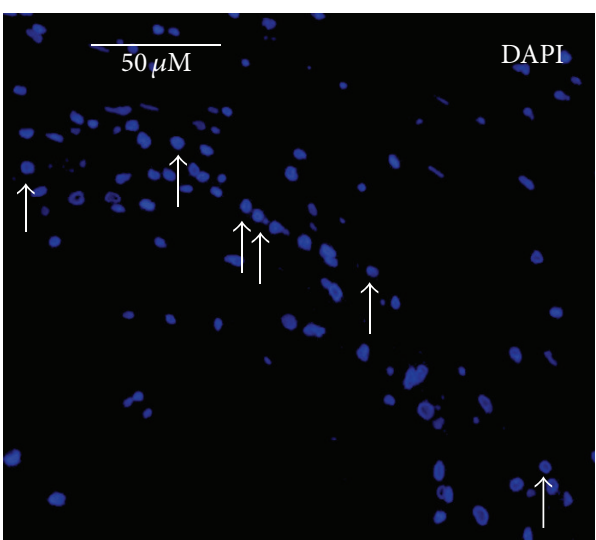

(a)

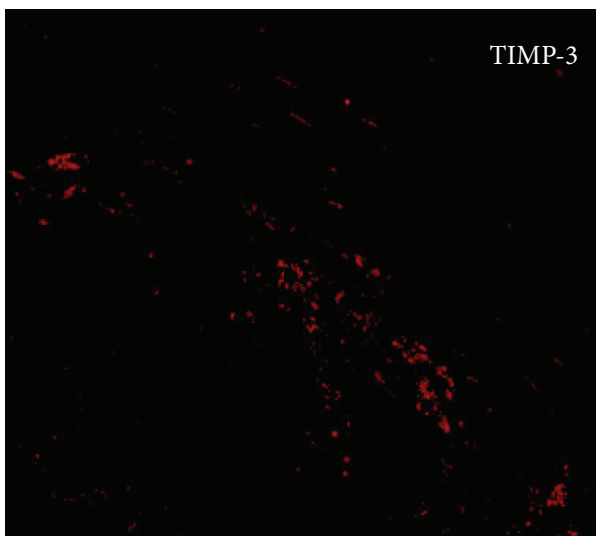

(c)

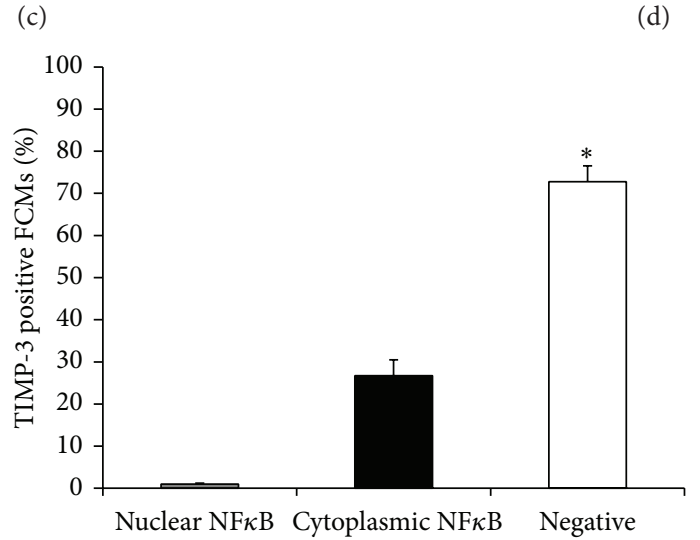

(e)

(b)

(d)
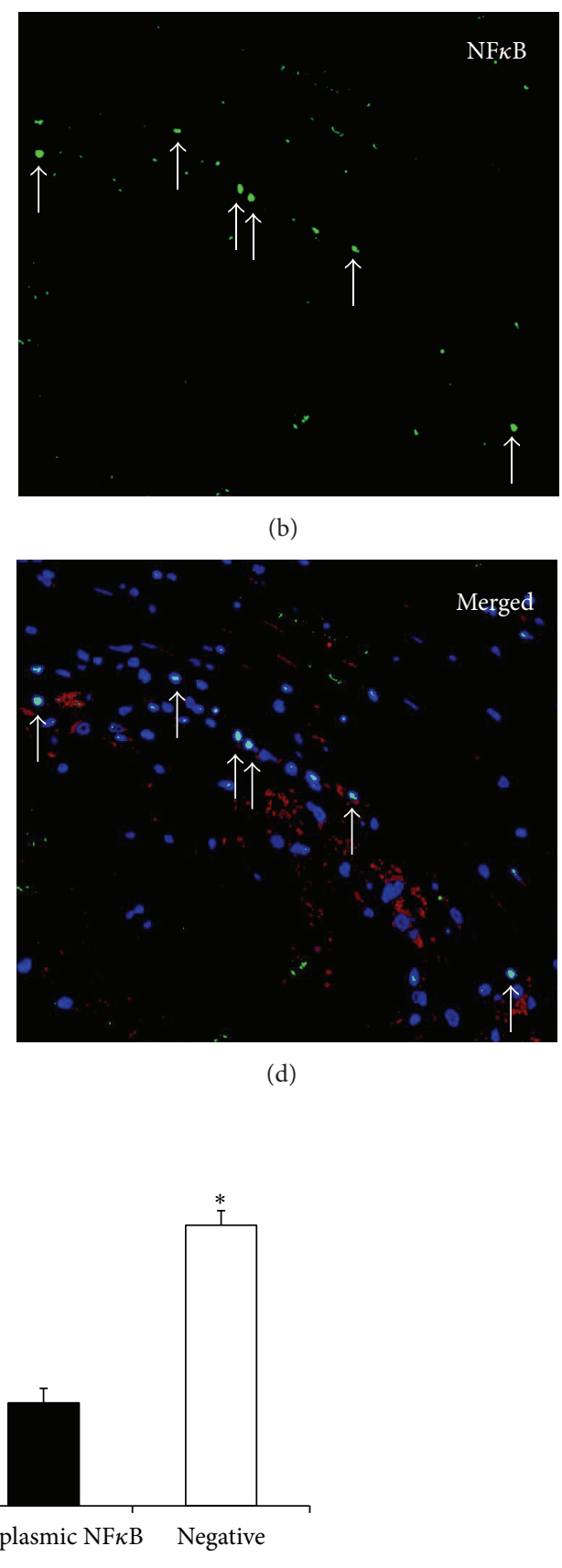

FIGURE 5: Immunohistochemical staining of carotid plaque sections for (a) 4',6-diamidino-2-phenylindole (DAPI), (b) NF $\kappa$ B, (c) TIMP3 , and (d) merged, demonstrating that the percentage of TIMP-3 positive FCMs with no nuclear NF $\kappa \mathrm{B}$ was significantly greater $(n=20$, $\left.{ }^{*} P<0.0001\right)$ compared to cytoplasmic or nuclear NF $\mathcal{K} B$ staining, as depicted in adjoining graph (e).

alternative activation, arginase-1. Hence foam-cell formation rather than $\mathrm{M} 1$ or M2 macrophage polarisation appears responsible for the MMP- $14^{\text {hi }}$ FCMs detected in advanced human atherosclerotic plaques.

By contrast to MMP-14, TIMP-3 mRNA was decreased during proinflammatory activation of macrophages or during foam-cell formation. A combination of these factors probably accounts for the TIMP- $3^{\text {lo }}$ populations seen in plaques. TIMP-3 positive macrophages/FCMs correlated and colocalised with the mannose receptor (MR), CD206. In agreement with other previous studies [38], we found that CD206 and TIMP-3 were highly expressed in macrophages differentiated in M-CSF (MM) and upregulated by IL-4 (MM2). However, TIMP-3 expression was only doubled after IL-4 treatment (Figure 1) and is therefore present in both phenotypes. MR positive macrophages/FCMs were previously reported to be mainly located away from the lipid core of plaques $[12,13]$, consistent with the location of 


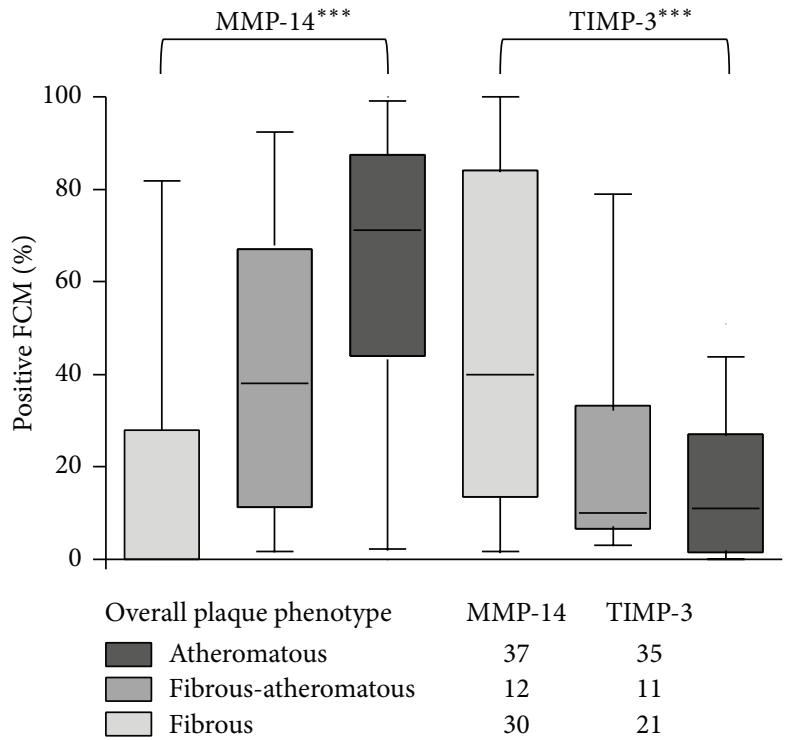

(a)

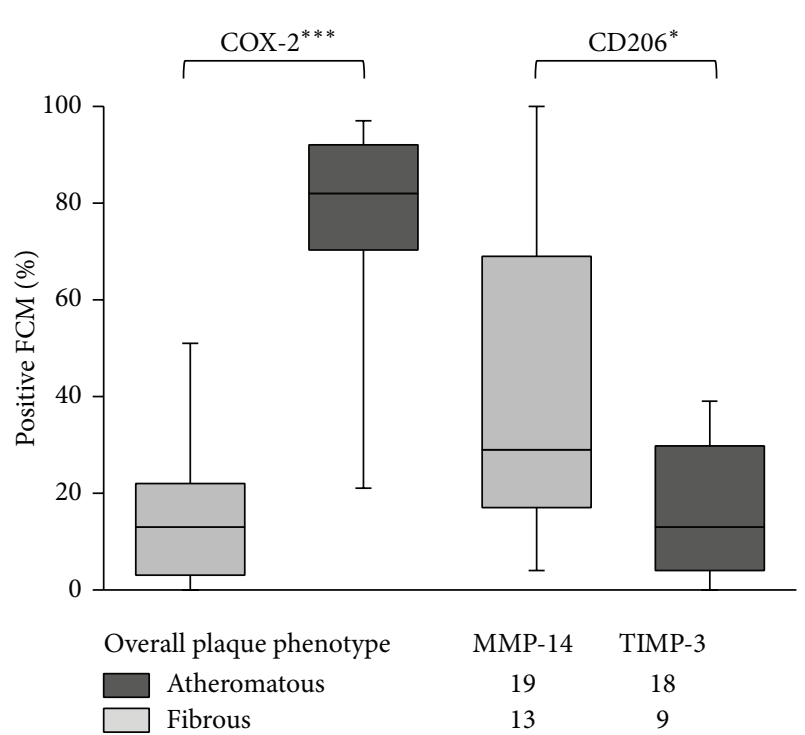

(b)

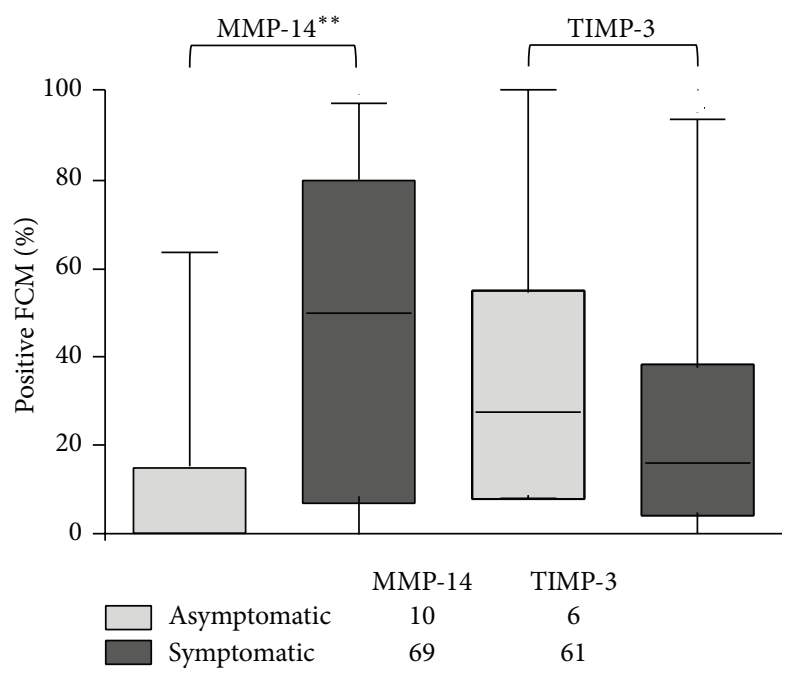

(c)

FIGURE 6: Relationship between MMP-14 and TIMP-3 staining and plaque morphology or symptoms. The percentage of CD68 positive FCMs that stained also for (a) MMP-14 and TIMP-3, or (b) COX-2 and CD206, was related to atheromatous, fibrous-atheromatous and fibrous carotid plaques. (c) Association of FCMs that stained also for MMP-14 and TIMP-3 was assessed in symptomatic and asymptomatic patients. Data analyzed using a Kruskal-Wallis test $\left({ }^{*} P<0.05,{ }^{* *} P<0.01,{ }^{* * *} P<0.001\right)$. Patient numbers within each group are depicted below each bar graph.

TIMP-3 positive macrophages/FCMs in our study. Although little is known about the regulation of TIMP-3 transcription [39], the striking decrease in protein expression we observed upon foam-cell formation is consistent with previous observations that posttranscriptional mechanisms can regulate TIMP expression during macrophage development [40]. Studies beyond the present scope are going on in our laboratories to investigate this possibility.

Injurious roles for MMP-14 and protective effects of TIMP-3 have been suggested from animal studies (see the Introduction). Consistent with these proposals, we found that MMP-14 positive FCM subpopulations were associated with histological features of plaque vulnerability in carotid plaques based primarily on correlating quantitative plaque characteristics derived from all the sections irrespective of histological appearance (Table 4). The conclusions were also confirmed in carotid atherosclerotic plaques by prior blinded assignment of lesions based on overall histological appearance (Figures 6 and 7). This second analysis can be criticised because it is based on subjective visual observation, although we have shown previously that there is good intraobserver agreement [23]. It is also evident that there is a gender imbalance between the differing plaque phenotypes which may act as a source of variability in the statistical analysis. Nonetheless, 

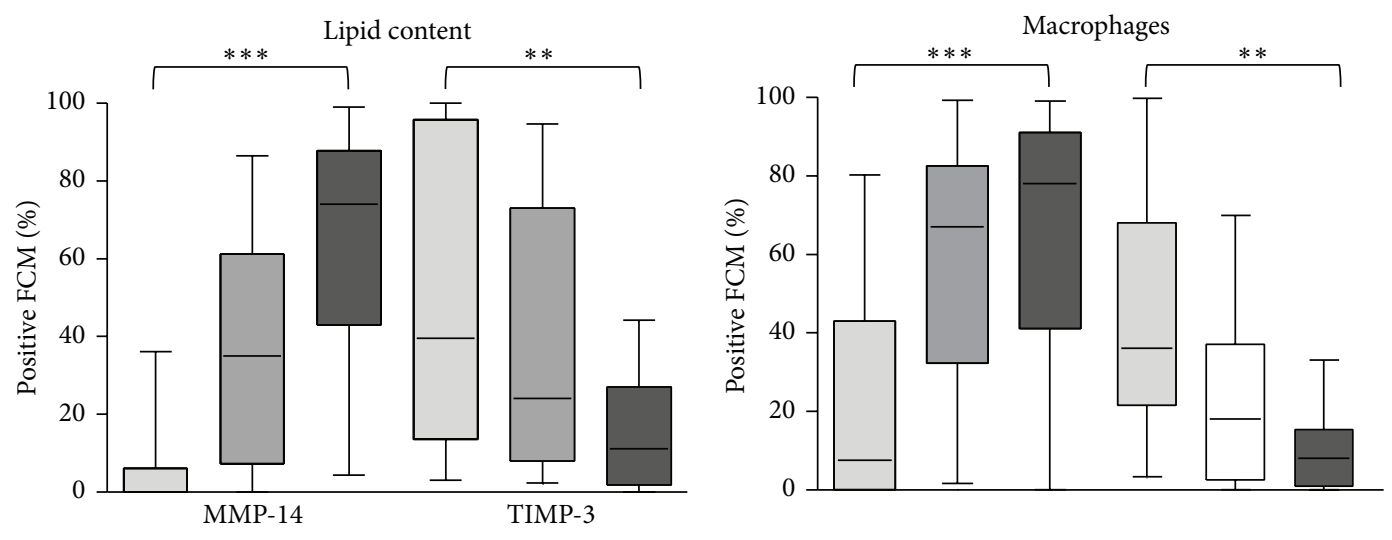

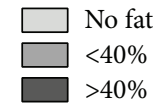

(a)
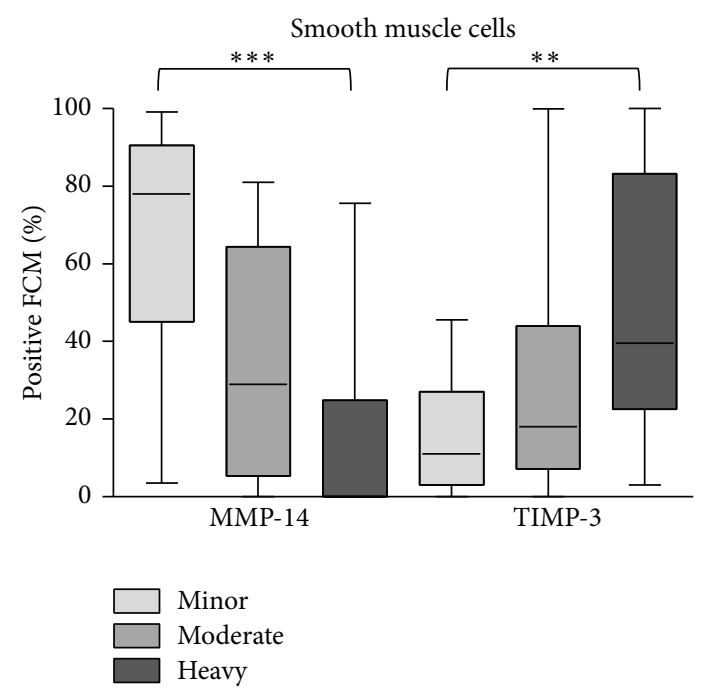

(c)

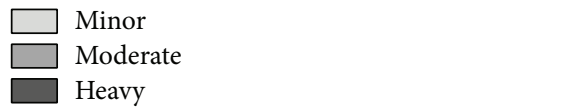

(b)

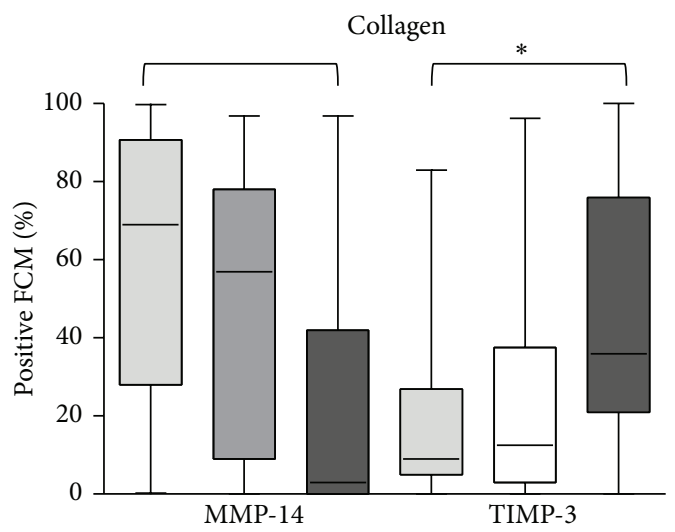

Minor

Moderate

Heavy

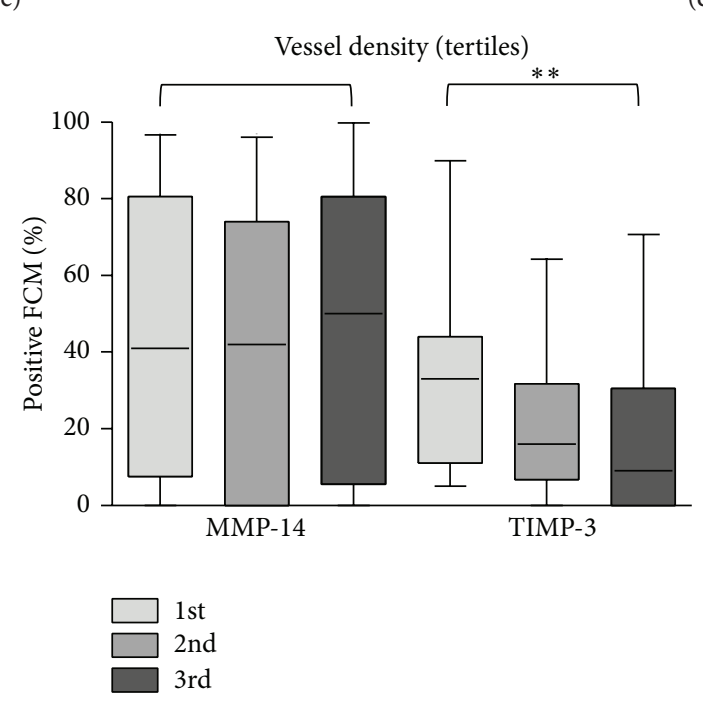

(d)

(e)

FIGURE 7: The percentage of MMP-14 and TIMP-3 positive foam-cell macrophages (median [95\% confidence limits]) was compared with semiquantitative plaque features and $P$ values computed with a Kruskal-Wallis test $\left({ }^{*} P<0.05,{ }^{* *} P<0.01,{ }^{* * *} P<0.001\right)$. 
TABle 4: Patient characteristics.

\begin{tabular}{|c|c|c|c|c|}
\hline Patient characteristics & $\begin{array}{l}\text { Fibrous plaques } \\
\quad(n=30)\end{array}$ & $\begin{array}{l}\text { Fibrous-atheromatous } \\
\qquad(n=12)\end{array}$ & $\begin{array}{l}\text { Atheromatous plaques } \\
\qquad(n=37)\end{array}$ & $P$ value \\
\hline Symptomatic patients $n(\%)$ & $22(73 \%)$ & $11(92 \%)$ & $36(97 \%)$ & $0.012^{*}$ \\
\hline Amaurosis fugax $n(\%)$ & $6(20 \%)$ & $5(42 \%)$ & $1(3 \%)$ & \multirow{3}{*}{$0.010^{*}$} \\
\hline TIA $n(\%)$ & $12(40 \%)$ & $4(33 \%)$ & $25(68 \%)$ & \\
\hline Stroke $n(\%)$ & $4(13 \%)$ & $2(17 \%)$ & $10(27 \%)$ & \\
\hline Asymptomatic $n(\%)$ & $8(27 \%)$ & $1(8 \%)$ & $1(3 \%)$ & \\
\hline $\mathrm{M}($ (ð)/F (ㅇ) & 11 đ 19 우 & 11 ô 1 ㅇ & 34 o 3 ㅇ & $0.007^{*}$ \\
\hline Age mean (sd) & $65(9.6)$ & $73(8.1)$ & $70(7.7)$ & $0.016^{*}$ \\
\hline Hypertension $n(\%)$ & $22(73 \%)$ & $10(83 \%)$ & $31(84 \%)$ & 0.540 \\
\hline Smoking $n(\%)$ & $9(30 \%)$ & $5(42 \%)$ & $13(35 \%)$ & 0.761 \\
\hline Diabetes $n(\%)$ & $5(17 \%)$ & $0(0 \%)$ & $4(11 \%)$ & 0.304 \\
\hline Hypercholesterolemia $n(\%)$ & $17(57 \%)$ & $6(50 \%)$ & $20(54 \%)$ & 0.924 \\
\hline Positive family history of heart disease & $7(23 \%)$ & $2(17 \%)$ & $10(27 \%)$ & 0.761 \\
\hline History of coronary intervention $n(\%)$ & $7(23 \%)$ & $3(25 \%)$ & $8(22 \%)$ & 0.967 \\
\hline History of peripheral intervention $n(\%)$ & $6(20 \%)$ & $1(8 \%)$ & $10(27 \%)$ & 0.379 \\
\hline History of myocardial infarction & $4(13 \%)$ & $3(25 \%)$ & $1(3 \%)$ & 0.064 \\
\hline Statins & $16(53 \%)$ & $5(42 \%)$ & $26(70 \%)$ & 0.147 \\
\hline Oral anticoagulants & $6(20 \%)$ & $4(33 \%)$ & $3(8 \%)$ & 0.098 \\
\hline Aspirin & $18(60 \%)$ & $3(25 \%)$ & $17(46 \%)$ & 0.114 \\
\hline Carbasalate calcium & $8(27 \%)$ & $8(67 \%)$ & $20(54 \%)$ & $0.023^{*}$ \\
\hline Aspirin or Carbasalate calcium & $24(80 \%)$ & $9(75 \%)$ & $34(92 \%)$ & 0.237 \\
\hline Dipyridamole & $18(60 \%)$ & $4(33 \%)$ & $22(60 \%)$ & 0.238 \\
\hline Clopidogrel & $3(10 \%)$ & $0(0 \%)$ & $7(19 \%)$ & 0.198 \\
\hline Diuretics & $9(30 \%)$ & $2(17 \%)$ & $11(30 \%)$ & 0.881 \\
\hline Beta blockers & $13(43 \%)$ & $3(25 \%)$ & $18(49 \%)$ & 0.355 \\
\hline Calcium antagonists & $6(20 \%)$ & $3(25 \%)$ & $12(32 \%)$ & 0.514 \\
\hline ACE inhibitors & $11(37 \%)$ & $3(25 \%)$ & $12(33 \%)$ & 0.764 \\
\hline Angiotensin II inhibitors & $5(17 \%)$ & $2(17 \%)$ & $4(11 \%)$ & 0.755 \\
\hline Insulin & $2(7 \%)$ & $0(0 \%)$ & $0(0 \%)$ & 0.187 \\
\hline Oral glucose inhibitors & $3(10 \%)$ & $0(0 \%)$ & $4(11 \%)$ & 1.000 \\
\hline
\end{tabular}

${ }^{*} P<0.05$.

TABLE 5: Correlations of immunostains with histological parameters for plaque vulnerability in human carotid plaques.

\begin{tabular}{|c|c|c|c|c|c|c|}
\hline \multirow{2}{*}{ Plaque characteristic } & \multicolumn{3}{|c|}{$\%$ of MMP- $14^{\text {hi }}$ FCMs } & \multicolumn{3}{|c|}{$\%$ of TIMP- $3^{\text {hi }}$ FCMs } \\
\hline & $n$ & rho & $P$ value & $n$ & rho & $P$ value \\
\hline Macrophage density & 79 & 0.453 & $<0.001$ & 68 & -0.316 & 0.009 \\
\hline SMC density & 76 & -0.466 & $<0.001$ & 66 & 0.283 & 0.021 \\
\hline$\%$ of MMP- $14^{\text {hi }}$ FCMs & $\mathrm{x}$ & $\mathrm{x}$ & $\mathrm{x}$ & 71 & -0.366 & 0.002 \\
\hline$\%$ of COX $-2^{+}$FCMs & 32 & 0.726 & $<0.001$ & 28 & -0.185 & 0.345 \\
\hline$\%$ of CD $206^{+}$FCMs & 27 & -0.013 & 0.948 & 27 & 0.606 & 0.001 \\
\hline
\end{tabular}

Correlations are shown between the percentage of CD68 positive foam-cell macrophages (FCMs) also positive for MMP-14 and TIMP-3 with quantitative histological markers and percentage of CD68 positive for COX-2 and CD206 using Spearman correlation tests. Negative correlations are shown in italics.

collectively our results demonstrate that MMP-14 positive FCMs associate with plaque vulnerability. Whereas TIMP-3 positive macrophages correlate with plaque stability.

In summary our findings show that FCMs in vulnerable atherosclerotic plaques can exhibit increased MMP14 and decreased TIMP-3 protein expression. This leads to heightened invasive capability, increased proliferation, and augmented susceptibility to apoptosis, as we have previously demonstrated [7]. Our work therefore suggests that reducing MMP-14 activity and increasing that of TIMP-3 could be valid therapeutic approaches to reduce plaque rupture and myocardial infarction. 
TABLE 6: MMP-14 and TIMP-3 expression and plaque characteristics.

\begin{tabular}{|c|c|c|c|c|c|}
\hline Plaque feature & Absent & $n$ & Present & $n$ & $P$ value \\
\hline \multicolumn{6}{|c|}{ MMP-14 (\% of positive foam cell macrophages) } \\
\hline Intraplaque haemorrhage & $24[0-64]$ & 51 & $67[28-81]$ & 28 & 0.007 \\
\hline Any thrombus or haemorrhage & $11[1-48]$ & 24 & $59[6-81]$ & 55 & 0.026 \\
\hline \multicolumn{6}{|c|}{ TIMP-3 (\% of positive foam cell macrophages) } \\
\hline Intraplaque haemorrhage & $24[8-43]$ & 44 & $11[5-25]$ & 24 & 0.095 \\
\hline Any thrombus or haemorrhage & $28[8-77]$ & 22 & $13[5-34]$ & 46 & 0.067 \\
\hline
\end{tabular}

The percentage of MMP-14 and TIMP-3 positive foam cell macrophages (median [95\% confidence limits]) was compared with semiquantitative plaque features and $P$ values computed with a Kruskal-Wallis test.

\section{Conflict of Interests}

The authors declare that there is no conflict of interests regarding the publication of this paper.

\section{Authors' Contribution}

Jason L. Johnson and Nicholas P. Jenkins contributed equally to this study. Jason L. Johnson and Andrew C. Newby designed the study. Jason L. Johnson, Wei-Chun Huang, Karina Di Gregoli, and Graciela B. Sala-Newby performed the cell culture studies and qPCR analysis. Jason L. Johnson, Nicholas P. Jenkins, and Vincent P. W. Scholtes performed the immunohistochemical staining and analysis. Jason $\mathrm{L}$. Johnson, Nicholas P. Jenkins, Vincent P. W. Scholtes, Frans L. Moll, and Gerard Pasterkamp participated in the collection of patient details, handling of human tissues, pathological evaluation, and phenotyping. Jason L. Johnson, Nicholas P. Jenkins, and Andrew C. Newby wrote the paper. All authors read and approved the final paper.

\section{Acknowledgments}

The authors acknowledge the skillful technical assistance of Ms Michelle Sommerville and Mrs Laura Bevan. The authors' work is supported by the British Heart Foundation (CH95/001, RG04/009, FS/07/053/24069) and the National Health Research Institute (UK), Bristol Biomedical Research Unit in Cardiovascular Medicine. W-C Huang held Fellowships from the Yen Tjing Ling Medical Foundation and Medical Foundation in Memory of Dr. Deh-Lin Cheng, Taiwan.

\section{References}

[1] R. Virmani, A. P. Burke, A. Farb, and F. D. Kolodgie, "Pathology of the vulnerable plaque," Journal of the American College of Cardiology, vol. 47, no. 8, pp. C13-C18, 2006.

[2] A. C. Newby, "Dual role of matrix metalloproteinases (matrixins) in intimal thickening and atherosclerotic plaque rupture," Physiological Reviews, vol. 85, no. 1, pp. 1-31, 2005.

[3] C. M. Dollery and P. Libby, "Atherosclerosis and proteinase activation," Cardiovascular Research, vol. 69, no. 3, pp. 625-635, 2006.

[4] A. C. Newby, "Matrix metalloproteinase inhibition therapy for vascular diseases.," Vascular pharmacology, vol. 56, no. 5-6, pp. 232-244, 2012.
[5] F. Schneider, G. K. Sukhova, M. Aikawa et al., "Matrix metalloproteinase-14 deficiency in bone marrow-derived cells promotes collagen accumulation in mouse atherosclerotic plaques," Circulation, vol. 117, no. 7, pp. 931-939, 2008.

[6] V. Casagrande, R. Menghini, S. Menini et al., "Overexpression of tissue inhibitor of metalloproteinase 3 in macrophages reduces atherosclerosis in low-density lipoprotein receptor knockout mice," Arteriosclerosis, Thrombosis, and Vascular Biology, vol. 32, no. 1, pp. 74-81, 2012.

[7] J. L. Johnson, G. B. Sala-Newby, Y. Ismail, C. M. Aguilera, and A. C. Newby, "Low tissue inhibitor of metalloproteinases 3 and high matrix metalloproteinase 14 levels defines a subpopulation of highly invasive foam-cell macrophages," Arteriosclerosis, Thrombosis, and Vascular Biology, vol. 28, no. 9, pp. 1647-1653, 2008.

[8] F. O. Martinez, L. Helming, and S. Gordon, "Alternative activation of macrophages: an immunologic functional perspective," Annual Review of Immunology, vol. 27, pp. 451-483, 2009.

[9] D. M. Mosser and J. P. Edwards, "Exploring the full spectrum of macrophage activation," Nature Reviews Immunology, vol. 8, no. 12, pp. 958-969, 2008.

[10] C. Monaco, S. M. Gregan, T. J. Navin, B. M. J. Foxwell, A. H. Davies, and M. Feldmann, "Toll-like receptor-2 mediates inflammation and matrix degradation in human atherosclerosis," Circulation, vol. 120, no. 24, pp. 2462-2469, 2009.

[11] A. C. Newby, S. J. George, Y. Ismail, J. L. Johnson, G. B. SalaNewby, and A. C. Thomas, "Vulnerable atherosclerotic plaque metalloproteinases and foam cell phenotypes," Thrombosis and Haemostasis, vol. 101, no. 6, pp. 1006-1011, 2009.

[12] M. A. Bouhlel, B. Derudas, E. Rigamonti et al., "PPAR $\gamma$ activation primes human monocytes into alternative $\mathrm{m} 2$ macrophages with anti-inflammatory properties," Cell Metabolism, vol. 6, no. 2, pp. 137-143, 2007.

[13] G. Chinetti-Gbaguidi, M. Baron, M. A. Bouhlel et al., "Human atherosclerotic plaque alternative macrophages display low cholesterol handling but high phagocytosis because of distinct activities of the PPAR $\gamma$ and LXR $\alpha$ pathways," Circulation Research, vol. 108, no. 8, pp. 985-995, 2011.

[14] J. L. Stöger, M. J. J. Gijbels, S. van der Velden et al., "Distribution of macrophage polarization markers in human atherosclerosis," Atherosclerosis, vol. 225, no. 2, pp. 461-468, 2012.

[15] A. V. Finn, O. Saeed, and R. Virmani, "Macrophage subsets in human atherosclerosis," Circulation Research, vol. 110, article e64, 2012.

[16] A. Kadl, A. K. Meher, P. R. Sharma et al., "Identification of a novel macrophage phenotype that develops in response to atherogenic phospholipids via Nrf2," Circulation Research, vol. 107, no. 6, pp. 737-746, 2010. 
[17] J. J. Boyle, H. A. Harrington, E. Piper et al., "Coronary intraplaque hemorrhage evokes a novel atheroprotective macrophage phenotype," The American Journal of Pathology, vol. 174, no. 3, pp. 1097-1108, 2009.

[18] J. J. Boyle, M. Johns, T. Kampfer et al., "Activating transcription factor 1 directs Mhem atheroprotective macrophages through coordinated iron handling and foam cell protection," Circulation Research, vol. 110, no. 1, pp. 20-33, 2012.

[19] A. V. Finn, M. Nakano, R. Polavarapu et al., "Hemoglobin directs macrophage differentiation and prevents foam cell formation in human atherosclerotic plaques," Journal of the American College of Cardiology, vol. 59, no. 2, pp. 166-177, 2012.

[20] B. A. N. Verhoeven, E. Velema, A. H. Schoneveld et al., "Athero-express: differential atherosclerotic plaque expression of mRNA and protein in relation to cardiovascular events and patient characteristics. Rationale and design," European Journal of Epidemiology, vol. 19, no. 12, pp. 1127-1133, 2004.

[21] J. P. G. Sluijter, W. P. C. Pulskens, A. H. Schoneveld et al., "Matrix metalloproteinase 2 is associated with stable and matrix metalloproteinases 8 and 9 with vulnerable carotid atherosclerotic lesions-a study in human endarterectomy specimen pointing to a role for different extracellular matrix metalloproteinase inducer glycosylation forms," Stroke, vol. 37, no. 1, pp. 235-239, 2006.

[22] D. P. V. de Kleijn, F. L. Moll, W. E. Hellings et al., "Local atherosclerotic plaques are a source of prognostic biomarkers for adverse cardiovascular events," Arteriosclerosis, Thrombosis, and Vascular Biology, vol. 30, no. 3, pp. 612-619, 2010.

[23] W. E. Hellings, G. Pasterkamp, A. Vollebregt et al., "Intraobserver and interobserver variability and spatial differences in histologic examination of carotid endarterectomy specimens," Journal of Vascular Surgery, vol. 46, no. 6, pp. 1147-1154, 2007.

[24] V. P. W. Scholtes, J. L. Johnson, N. Jenkins et al., "Carotid atherosclerotic plaque matrix metalloproteinase-12-positive macrophage subpopulation predicts adverse outcome after endarterectomy," Journal of the American Heart Association, vol. 1, no. 6, Article ID e001040, 2012.

[25] E. Monsalve, A. Ruiz-García, V. Baladrón et al., "Notch1 upregulates LPS-induced macrophage activation by increasing NF- $\kappa$ B activity," European Journal of Immunology, vol. 39, no. 9, pp. 2556-2570, 2009.

[26] F. O. Martinez, S. Gordon, M. Locati, and A. Mantovani, “Transcriptional profiling of the human monocyte-to-macrophage differentiation and polarization: new molecules and patterns of gene expression," Journal of Immunology, vol. 177, no. 10, pp. 7303-7311, 2006.

[27] D. Shiffman, T. Mikita, J. T. N. Tai et al., "Large scale gene expression analysis of cholesterol-loaded macrophages," The Journal of Biological Chemistry, vol. 275, no. 48, pp. 3732437332, 2000.

[28] S.-C. Sun, P. A. Ganchi, D. W. Ballard, and W. C. Greene, "NF- $\kappa$ B controls expression of inhibitor $\mathrm{I} \kappa \mathrm{B} \alpha$ : evidence for an inducible autoregulatory pathway," Science, vol. 259, no. 5103, pp. 19121915, 1993.

[29] K. di Gregoli, N. Jenkins, R. Salter, S. White, A. C.. Newby, and J. L. Johnson, "Microrna-24 regulates macrophage behavior and retards atherosclerosis," Arteriosclerosis, Thrombosis, and Vascular Biology, 2014.

[30] A. Mantovani, C. Garlanda, and M. Locati, "Macrophage diversity and polarization in atherosclerosis: a question of balance," Arteriosclerosis, Thrombosis, and Vascular Biology, vol. 29, no. 10, pp. 1419-1423, 2009.
[31] A. C. Thomas, G. B. Sala-Newby, Y. Ismail, J. L. Johnson, G. Pasterkamp, and A. C. Newby, "Genomics of foam cells and nonfoamy macrophages from rabbits identifies arginase-I as a differential regulator of nitric oxide production," Arteriosclerosis, Thrombosis, and Vascular Biology, vol. 27, no. 3, pp. 571-577, 2007.

[32] W. Peeters, W. E. Hellings, D. P. V. de Kleijn et al., "Carotid atherosclerotic plaques stabilize after stroke: insights into the natural process of atherosclerotic plaque stabilization," Arteriosclerosis, Thrombosis, and Vascular Biology, vol. 29, no. 1, pp. 128-133, 2009.

[33] G. Chinetti-Gbaguidi and B. Staels, "Macrophage polarization in metabolic disorders: functions and regulation," Current Opinion in Lipidology, vol. 22, no. 5, pp. 365-372, 2011.

[34] J. L. Johnson and A. C. Newby, "Macrophage heterogeneity in atherosclerotic plaques," Current Opinion in Lipidology, vol. 20, no. 5, pp. 370-378, 2009.

[35] W.-C. Huang, G. B. Sala-Newby, A. Susana, J. L. Johnson, and A. C. Newby, "Classical macrophage activation up-regulates several matrix metalloproteinases through mitogen activated protein kinases and nuclear factor- $\kappa$ B," PLoS ONE, vol. 7, no. 8, Article ID e42507, 2012.

[36] B. Reel, G. B. Sala-Newby, W. Huang, and A. C. Newby, "Diverse patterns of cyclooxygenase-independent metalloproteinase gene regulation in human monocytes," British Journal of Pharmacology, vol. 163, no. 8, pp. 1679-1690, 2011.

[37] J. Spann Nathanael, X. Garmire Lana, G. McDonald Jeffrey et al., "Regulated accumulation of desmosterol integrates macrophage lipid metabolism and inflammatory responses," Cell, vol. 151, pp. 138-152, 2012.

[38] R. P. Fabunmi, G. K. Sukhova, S. Sugiyama, and P. Libby, "Expression of tissue inhibitor of metalloproteinases-3 in human atheroma and regulation in lesion-associated cells: a potential protective mechanism in plaque stability," Circulation Research, vol. 83, no. 3, pp. 270-278, 1998.

[39] M. Wick, R. Haronen, D. Mumberg et al., "Structure of the human TIMP-3 gene and its cell cycle-regulated promoter," Biochemical Journal, vol. 311, no. 2, pp. 549-554, 1995.

[40] G. A. R. Doyle, U. K. Saarialho-Kere, and W. C. Parks, "Distinct mechanisms regulate TIMP-1 expression at different stages of phorbol ester-mediated differentiation of U937 cells," Biochemistry, vol. 36, no. 9, pp. 2492-2500, 1997. 


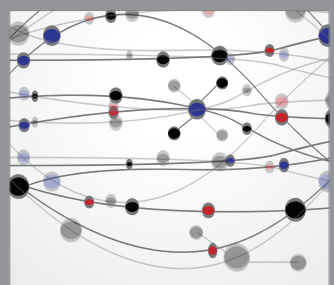

The Scientific World Journal
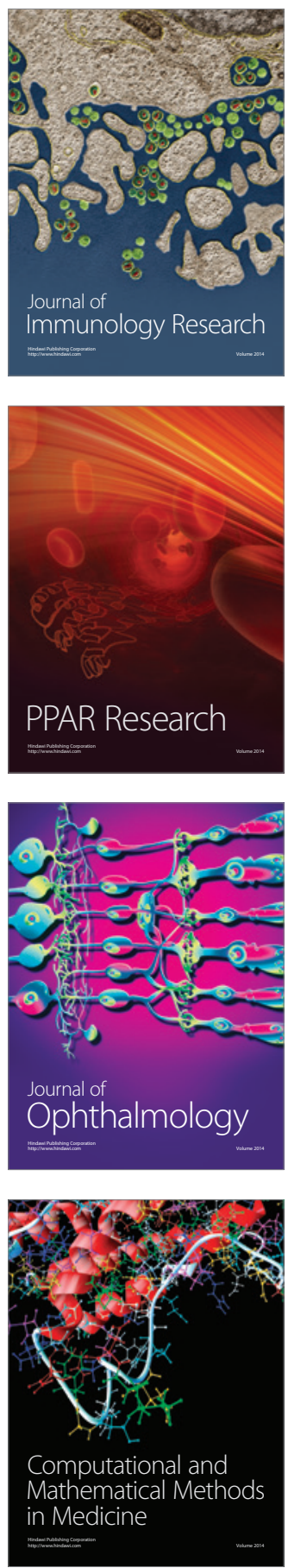

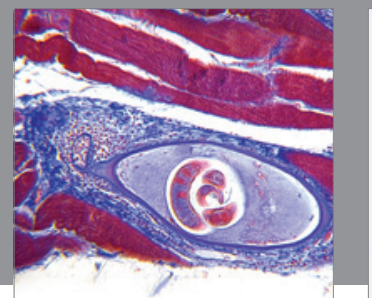

Gastroenterology

Research and Practice
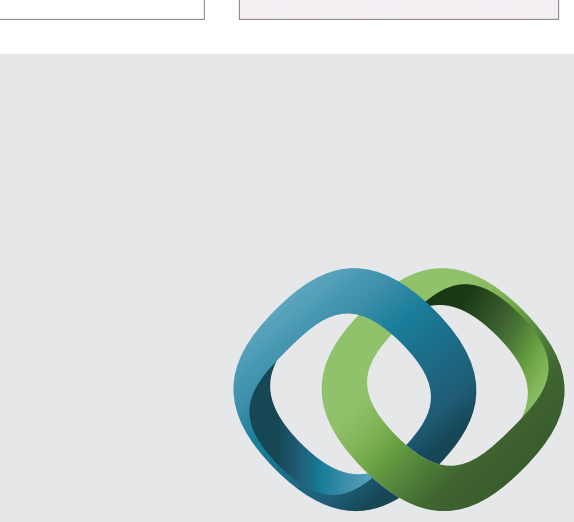

\section{Hindawi}

Submit your manuscripts at

http://www.hindawi.com
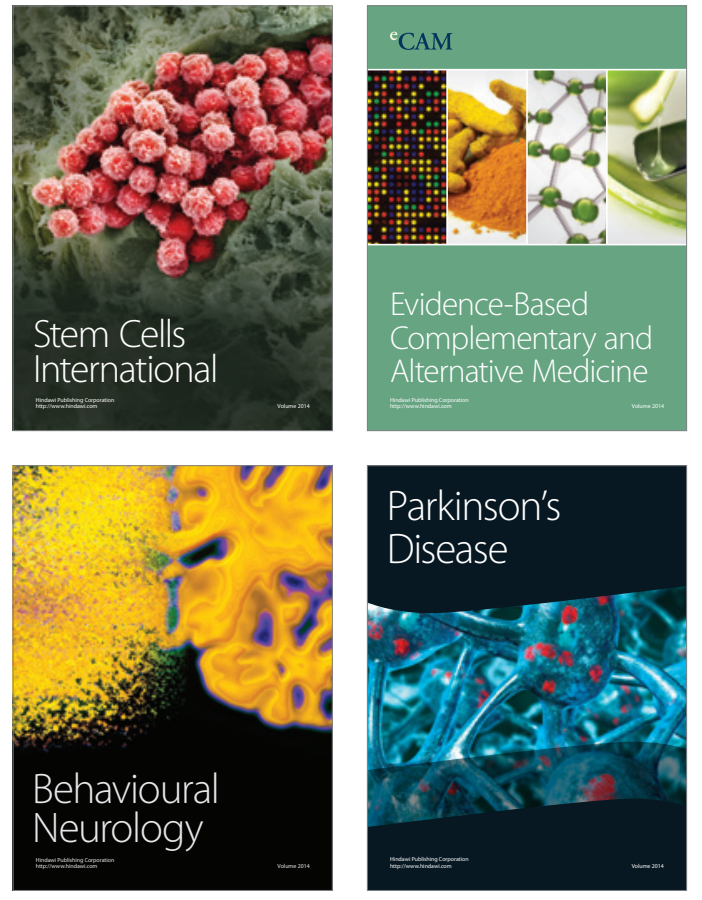
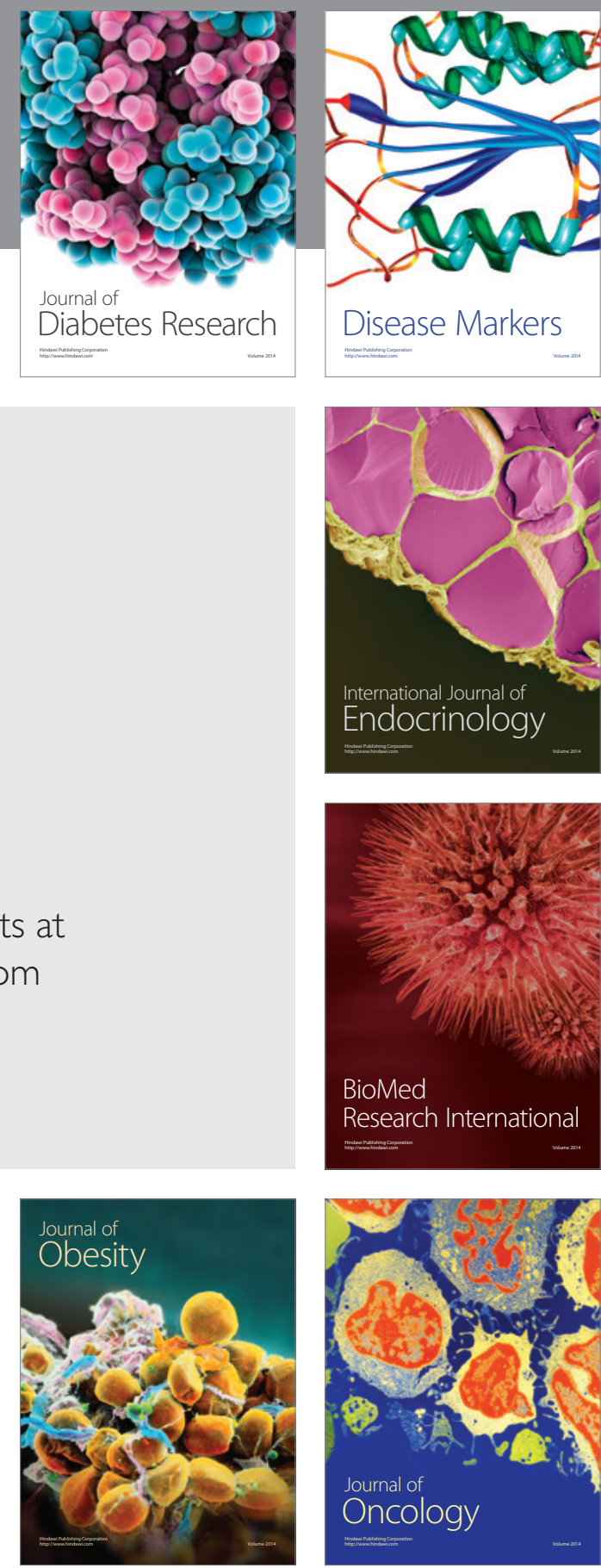

Disease Markers
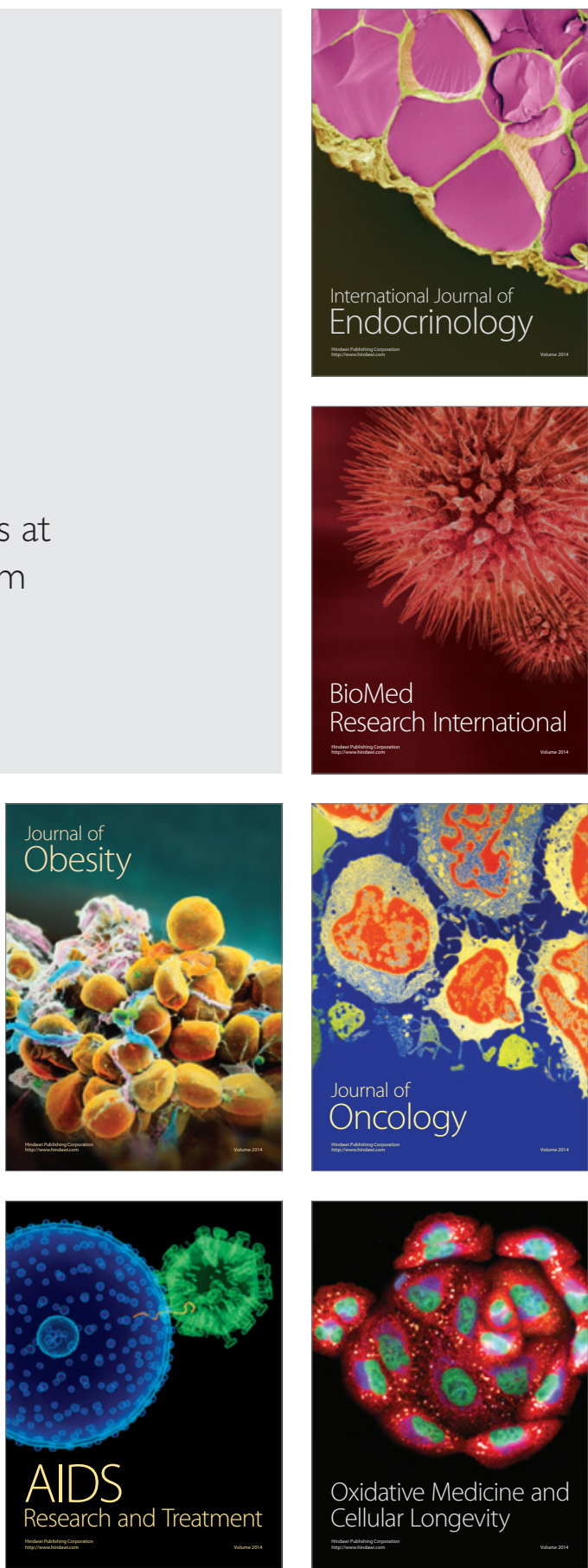